\title{
Remédiation aux erreurs communes de l'écriture académique chez les étudiants de magistère de la faculté de pédagogie de Minia (didactique du FLE) à la lueur de l'approche des processus d'écriture
}

\author{
Mohamed Gomaa Refaï Ibrahim*
}

\section{Résumé :}

L'écriture académique est le moyen de présenter les études et les résultats des recherches scientifiques dans tous les domaines de la science. Cette écriture a ses caractéristiques et son style spécifique. Les étudiants universitaires ont besoins toujours d'améliorer leurs compétences en écriture académiques et de corriger leurs erreurs dans ce domaine. Cette recherche a visé à déterminer les erreurs communes en écriture académique chez les étudiants de magistère (didactique du FLE). Pour atteindre ce but, le chercheur a élaboré un questionnaire qui a été soumis à 10 membres du corps enseignant en didactique du FLE. De même, le chercheur a élaboré et administré un test diagnostique pour déterminer les erreurs communes en écriture académique auprès un échantillon des étudiants de magistère de la faculté de pédagogie de Minia (didactique du FLE). Cette recherche a, également, visé à remédier aux erreurs communes en écriture académique chez les étudiants de magistère de la faculté de pédagogie de Minia (didactique du FLE). Pour atteindre ce but, le chercheur a élaboré un programme basé sur la stratégie des processus d'écriture. La partie pratique de notre programme se compose de ₹ séquences : Génération des idées, Lecture et Prise des notes, Faire un brouillon, L'évaluation (Evaluation par les pairs - Evaluation par le formateur) et Réécriture. L'expérimentation de notre programme a eu lieu en vacances d'été pendant les deux mois de juillet et d'aout. Un pré/post-test a été administré avant et après l'expérimentation du programme auprès des participants (10 participants des étudiants de magistère de la faculté de pédagogie de Minia (didactique du FLE)). Les résultats ont montré d'il y a des différences significatives entre la moyenne des notes des participants dans le pré/test et le post/test de l'écriture académique en faveur du post test. Ces résultats montrent l'efficacité du programme proposé basé sur la stratégie des processus d'écriture dans la remédiation aux erreurs communes en écriture académique chez les étudiants de magistère de la faculté de pédagogie de Minia (didactique du FLE). Le chercheur recommande, alors, d'utiliser la stratégie des processus d'écriture dans l'enseignement de l'écriture académique chez les étudiants des études supérieures.

Mots-clés : écriture académique - approche des processus d'écriture - erreurs communes didactique du FLE

\section{Introduction :}

L'écriture est une activité d'une importance primordiale car elle est le fruit de toutes les autres activités de la langue surtout la compréhension écrite et la compréhension orale. C'est à travers l'écriture qu'on peut enregistrer les idées, les données, les informations, ... . De même,

'Maître de conférences en didactique du FLE Faculté de pédagogie - Université de Minia.

Email: mgomaa660@gmail.com 
l'écriture est, toujours, utilisée par les scientifiques pour rédiger les rapports et les résultats de leurs recherches ; c'est ce qu'on appelle l'écriture scientifique ou l'écriture académique.

L'écriture scientifique ou «l'écriture académique est un système de représentation qui rend partageable un savoir disciplinaire pour ses membres [...] (Elle) correspond, en réalité, à un « langage » académique, qui est une langue véhiculaire ayant un caractère composite, d'un côté protocolaire et d'un autre côté hautement spécialisé » (Ito, 2015, p. 58).

Autrement dit, l'écriture académique est le moyen utilisé par les universitaires, qu'ils soient un corps enseignant, des étudiants en premier degré, des étudiants des études supérieures, pour rédiger et partager leurs recherches scientifiques, leurs articles académiques, et tous leurs produits scientifiques et académiques.

L'écriture académique concerne le français sur objectifs universitaires (FOU). Celui-ci est assimilé à du français sur objectifs spécifiques (FOS) destiné à des publics d'étudiants qui doivent suivre leurs études dans un système universitaire. Le FOS est, en effet, une méthodologie, centrée sur l'identification précise des besoins, que l'on peut établir de manière fiable pour des publics professionnels dont on cerne les métiers et les situations de travail. Dans le FOU, la didactique du français langue étrangère change de point de focalisation de manière officielle : il ne s'agit plus de concevoir un programme centré sur l'apprenant mais sur une situation; c'est être étudiant dans un système universitaire (Mourlhon-Dallies, 2011).

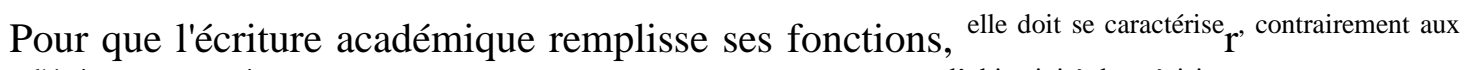
autres types d'écriture, par certaines caractéristiques spécifiques telles que l'objectivité, la précision, la concision,

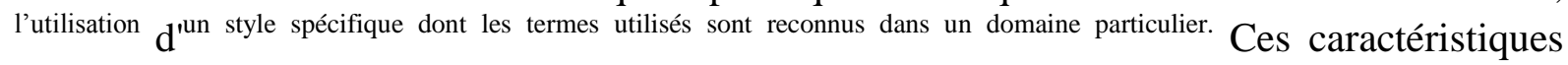
rendent le style plus simple et le message plus compréhensible.

Cependant, les étudiants universitaires révèlent un grand nombre de déséquilibres qui affectent de nombreux aspects de leurs écrits de recherche. Il est reconnu que les compétences de la rédaction académique ne sont pas seulement linguistiques ou méthodologiques ; mais on doit prendre en considération sa fonction cognitive et ses trois dimensions réflexive, argumentative et dialogique (Mroue, 2014).

Plakhotnik et Rocco (2012) constatent que la plupart des étudiants arrivent à leurs programmes d'études supérieures avec des compétences en écriture académique insuffisantes pour réussir et exceller dans leurs études [traduction libre, p.76] . De même, Donahue (2008) déclare que les étudiants rencontrent quelques difficultés dans les écrits théoriques surtout dans l'introduction, le développement d'une problématique, la reformulation des textes théoriques, les citations, la restitution d'un cadre théorique et le résumé. Ferraris \& Hernández (2015) ont indiqué, également, que les parties les plus problématiques à rédiger dans les processus d'écriture au niveau du contenu sont : le résumé, l'introduction et la conclusion.

Afin de déterminer les difficultés de l'écriture académique, Bou et Cavalla (2011) ont mené une enquête au moyen d'un questionnaire électronique (52 questions majoritairement fermées) diffusé aux enseignants-chercheurs. Parmi les variables du questionnaire étaient les difficultés discursives, méthodologiques et d'apprentissage. Les difficultés génériques et spécifiques sont présentées ainsi : 
- Difficultés générales à l'écrit : difficultés discursives (nuancer le discours $(47,5 \%)$ argumenter (38\%) - démontrer/ expliquer (35\%) - enchaîner et relier des idées (32,5\%) - utiliser la terminologie adéquate (25\%)), difficultés méthodologiques (présenter une problématique $(42,5 \%)$ - synthétiser l'information (36\%) - introduire/ conclure (33\%) respecter des normes d'écriture (30,5\%) - produire une rhétorique efficace (30\%)) ;

- Difficultés spécifiques à l'écrit : difficultés en sciences expérimentales et techniques (présenter des résultats (24\%) - définir/ caractériser (19\%) - raconter des faits/ événements $(11 \%)$ ), difficultés en sciences humaines et sociales (respecter un plan $(16 \%)$ - mettre en relief $(11 \%)$ - citer un auteur (7.5\%)).

Dans cette perspective, Reuter (2004) détermine sept dysfonctionnements de l'écriture académique chez les étudiants de master, ce sont les suivants :

- Les imprécisions : la formulation des concepts, des questions, des hypothèses, des critères ;

- Des modalités énonciatives inadaptées : assertions sous forme de vérités générales (il faut / on doit / il est nécessaire de...) ;

- L'absence de fonctionnalisation de certains passages ;

- Une tendance à la juxtaposition qui fait l'impasse sur l'explicitation des articulations entre les composantes et qui peut se manifester à différents niveaux d'organisation de l'écrit : au niveau structurel avec la juxtaposition des parties (notamment théorique et empirique) ;

- Des déséquilibres, qu'exprime souvent la longueur des passages textuels (soit aux théories par rapport au travail empirique, soit l'inverse) ;

- Des dysfonctionnements dans l'insertion du discours d'autrui ;

- Des problèmes de lisibilité repérables notamment au travers de faits textuels ;

Dans les dernières années, les chercheurs se sont préoccupés pour développer les compétences de l'écriture académique ; l'étude d'Al-Fadda, (2012) a examiné les effets de l'utilisation des wikis pour développer les performances écrites (écriture académique) des étudiants d'anglais langue étrangère. Les participants étaient des étudiants du programme d'année préparatoire en anglais (de niveau avancé) dans une université privée à Riyadh, en Arabie saoudite. L'étude a utilisé la méthode quasi-expérimentale, avec deux groupes expérimental et contrôle de 40 étudiants. Les résultats ont affirmé l'efficacité des wikis pour améliorer les compétences en écriture des étudiants de l'université.

L'étude de Sapkota (2013) a visé le développement des compétences en écriture à travers l'utilisation de la technique de correction par les pairs et les enseignants. La technique de correction par les pairs et de correction des enseignants s'est révélée productive dans l'enseignement de l'écriture par la recherche-action dans son ensemble.

L'étude d'Alqiawi (2015) a élaboré un modèle pour remédier aux difficultés en écriture académique des étudiants universitaires en proposant un modèle basé sur les stratégies de la pensée critique. Ce modèle est divisé en trois parties correspondantes aux caractéristiques principales d'un article académique. Il couvre le cadre externe du document académique, l'analyse et la persuasion tout au long du document, des suggestions d'auto-évaluation de 
l'essai écrit et les directives générales. Les résultats montrent que le modèle a atteint un niveau acceptable de validité et de fiabilité et peut être utilisé pour former des étudiants universitaires à la rédaction d'essais universitaires.

L'étude de Hussien et Elmahlawy (2018) a vérifié l'efficacité d'un site électronique d'apprentissage basé sur la théorie de l'apprentissage basé sur le cerveau pour remédier aux difficultés de l'écriture académique en langue arabe et les stratégies de l'apprentissage autorégulé chez les étudiants du diplôme spécial de la faculté de pédagogie d'Alexandrie.

On remarque que l'intérêt à l'écriture académique augmente d'un jour à un autre puisque le besoin des recherches académiques rigides augmente. Certaines études antérieures se sont intéressé à déterminer les difficultés de l'écriture académique. D'autres études antérieures ont visé à remédier à ces difficultés. De même, certaines études antérieures ont visé à développer les compétences de l'écriture académique chez les étudiants universitaires.

Dans les années 1980, l'écriture est passée d'une approche basée sur la langue à une approche basée sur les processus. Les processus d'écriture est une approche dans laquelle les apprenants de langue se concentrent sur les processus selon lesquels ils produisent leurs écrits plutôt que sur les produits eux-mêmes. Toutefois, les apprenants ont sûrement besoin de compléter leurs produits, mais le processus d'écriture lui-même est davantage souligné. En se concentrant sur les processus d'écriture, les apprenants en viennent à mieux se comprendre et à apprendre à travailler à travers l'écriture. Ils peuvent explorer quelles stratégies sont conformes à leur style d'apprentissage (Onozawa, 2010) [traduction libre, p. 156].

Reconnaître l'écriture en tant que processus implique de comprendre l'écriture comme une série d'actions et de considérer l'effort d'écriture dans son intégralité : pré-écriture, écriture et réécriture. L'écriture, dans cette approche est considérée comme une interaction entre l'étudiant écrivain, le texte et le formateur. Celui-ci devient alors un facilitateur plutôt qu'un juge (Barnett, 1992) [traduction libre, p. 18].

Par conséquent, selon cette approche, les étudiants écrivains se préoccupent des processus de l'écriture tels que la détermination des idées, la prise des notes (dans le cas d'utilisation des références), l'écriture d'un brouillon, la reformulation du texte, la liaison entre les phrases et les paragraphes, etc. Ces processus sont complétés par des processus d'évaluation et d'autoévaluation qui sont basés sur des caractéristiques spécifiques de l'écriture. Autrement dit, cette stratégie met l'accent sur les processus d'écriture avec un intérêt complémentaire de l'écriture en tant que produit.

Brown (2001) déclare qu'une approche des processus est importante pour les apprenants de langues car ils sont les créateurs de leurs propres textes ; leurs motivations intrinsèques sont ensuite valorisées. En d'autres termes, cette approche permet aux étudiants de gérer leur propre travail en leur donnant la possibilité de réfléchir tout en écrivant.

L'avantage de l'approche des processus est qu'il est possible de combiner plusieurs approches et d'intégrer d'autres compétences. Afin de rendre la classe d'écriture plus efficace, les enseignants prennent en compte divers aspects, tels que les compétences de chaque étudiant et ses besoins. Par exemple, certains étudiants peuvent avoir besoin de se concentrer 
sur les pratiques au niveau de la phrase ou d'apprendre à organiser leurs idées de manière logique (Onozawa, 2010) [traduction libre, p. 157].

Plusieurs études antérieures ont affirmé l'efficacité de l'approche des processus d'écriture dans le développement des compétences de l'écriture chez les apprenants (Omari, 2005 ; Ho, 2006 ; Sun \& Feng, 2009 ; Bayat, 2014 ; Alodwan et Ibnian, 2014).

L'étude d'Omari (2005) a visé à examiner les effets de l'utilisation de la méthode des processus d'écriture sur les résultats en écriture des étudiants d'anglais langue seconde de dixième année en Jordanie. Une école élémentaire de base a été choisie au hasard. Une section de dixième année a appris à écrire en utilisant la méthode des processus, tandis que l'autre section a été enseignée en utilisant une approche traditionnelle. Les résultats ont montré que les élèves à qui on a appris à utiliser la méthode des processus d'écriture ont obtenu des résultats significativement plus élevés que ceux à qui on a appris à utiliser l'approche traditionnelle en ce qui concerne leur rendement écrit.

L'étude de Ho (2006) a examiné l'efficacité des processus d'écriture pour améliorer les compétences en écriture et l'attitude envers l'écriture en anglais chez les élèves du premier et du deuxième cycle primaire. Les résultats ont révélé que le programme basé sur l'approche du processus d'écriture avait produit des résultats positifs dans toutes les classes et aux niveaux supérieur et inférieur.

L'étude expérimentale de Sun \& Feng (2009) a visé à utiliser l'approche des processus d'écriture pour développer les compétences d'écriture chez les apprenants d'anglais langue seconde. Les résultats de cette étude a montré que les l'approche des processus d'écriture a aidé les apprenants d'anglais langue seconde à faire des progrès significatifs dans leurs compétences en écriture.

L'étude de Bayat (2014) a examiné l'effet de l'approche des processus d'écriture sur le succès de l'écriture (un essai académique) et l'anxiété concernant l'écriture. Les participants à cette étude étaient des élèves d'enseignement préscolaire de première année ; une méthode quasi expérimentale avec un pré/post-test a été utilisée. À la suite de l'analyse statistique, l'étude a révélé que l'approche des processus d'écriture avait une efficacité significative dans le succès de l'écriture et l'apaisement de l'anxiété concernant l'écriture.

L'étude d'Alodwan et Ibnian (2014) a visé à examiner l'effet de l'utilisation de l'approche des processus d'écriture sur le développement des compétences de la rédaction d'essais universitaires. Les résultats de l'étude ont montré que l'approche des processus d'écriture avait eu une incidence positive sur les compétences en rédaction d'essais en anglais langue étrangère des étudiants. Sur la base des résultats de l'étude, les chercheurs ont recommandé de mettre davantage l'accent sur l'enseignement de l'écriture en tant que processus. Les chercheurs ont également recommandé de mettre davantage l'accent sur l'étape de pré-écriture en raison de son rôle essentiel dans le processus d'écriture.

L'intégration entre l'approche des processus d'écriture et l'approche de l'écriture en tant que produit a été utilisé par Jubran (2017) pour développer l'écriture chez les apprenants jordaniens d'anglais langue étrangère à l'université appliquée Al-Balqa. Le chercheur a adopté une approche éclectique de l'enseignement de l'écriture en synthétisant la force des 
deux approches de l'écriture. L'échantillon (90 apprenants débutants) était divisé en trois groupes : un groupe expérimental et deux groupes témoins. Les résultats de l'étude indiquent qu'il existe des différences significatives dans le post-test entre les deux groupes témoins et le groupe expérimental en faveur du groupe expérimental.

De même, Zabo (2017) déclare que l'écriture académique s'apprend à travers l'écriture et la réécriture en effaçant, en modifiant et en écrivant, encore et encore.... L'approche des processus de l'écriture permet d'écrire, de réécrire, de réviser et de modifier ce qu'on écrit.

\section{Problématique de la recherche :}

Le chercheur a observé pendant l'enseignement (et la direction des recherches) aux étudiants de la faculté de pédagogie, université de Minia que les étudiants de magistère commettent beaucoup d'erreurs dans leurs écritures académiques. Ce problème est vérifié à travers la compulsation des plans présentés par les étudiants pour l'inscription au grade de magistère, la compulsation de quelques thèses de magistères présentés dans le domaine de la didactique du FLE. De même, un rapport, élaboré par le département du curricula et de méthodologie (faculté de pédagogie de Minia) en 2018, indique que les étudiants de magistère en didactique du FLE passent beaucoup plus de temps dans l'élaboration de leurs thèses en comparaison avec leurs collègues dans les autres sections. Le chercheur a fait des entretiens avec les membres du corps enseignant en didactique du FLE à la faculté de pédagogie de Minia qui ont tous affirmé que les étudiants de magistère font beaucoup d'erreurs dans leurs écritures académiques. Reuter (2004) déclare que l'écriture de recherche ne fait pas l'objet d'un véritable enseignement malgré les problèmes constatés. Les étudiants se retrouvent donc sans grande préparation, en situation de passer d'une posture de récepteur de savoirs à une autre de producteur de savoirs, de changer de normes et de genres d'écrits. Pour envisager ce problème, la recherche actuelle va répondre aux questions suivantes :

1. Quelles sont les erreurs communes de l'écriture académique chez les étudiants de magistère de la faculté de pédagogie de Minia (didactique du FLE) ?

2. Quel est le programme proposé basé sur l'approche des processus d'écriture pour remédier aux erreurs communes de l'écriture académique chez les étudiants de magistère de la faculté de pédagogie de Minia (didactique du FLE) ?

3. Quelle est l'efficacité du programme proposé basé sur l'approche des processus d'écriture pour remédier aux erreurs communes de l'écriture académique chez les étudiants de magistère de la faculté de pédagogie de Minia (didactique du FLE) ?

\section{Objectifs de la recherche :}

1. Déterminer une liste d'erreurs communes de l'écriture académique chez les étudiants de magistère de la faculté de pédagogie de Minia (didactique du FLE) ;

4. Remédier aux erreurs communes de l'écriture académique chez les étudiants de magistère de la faculté de pédagogie de Minia (didactique du FLE); 
5. Vérifier l'efficacité d'un programme proposé basé sur l'approche des processus d'écriture pour remédier aux erreurs communes de l'écriture académique chez les étudiants de magistère de la faculté de pédagogie de Minia (didactique du FLE).

\section{Délimitation de la recherche :}

Cette recherche se délimite à :

1. Un échantillon d'étudiants qui sont inscrits au grade de magistère à la faculté de pédagogie de Minia (didactique du FLE) car ils ont, déjà, étudié les matières qui ont pour objectifs de développer leurs compétences méthodologiques.

2. Quelques erreurs communes de l'écriture académique les plus importantes (erreurs lexicales, erreurs grammaticales et erreurs syntaxiques) chez les étudiants de magistère de la faculté de pédagogie de Minia (didactique du FLE).

\section{Hypothèses de la recherche :}

1. Il y a une différence significative entre la moyenne des notes des étudiants du groupe expérimental au pré-post/test de l'écriture académique chez les étudiants de magistère de la faculté de pédagogie de Minia (didactique du FLE) en faveur du post/test.

a) Il y a une différence significative entre la moyenne des notes des étudiants $\mathrm{du}$ groupe expérimental au pré-post/test de l'écriture académique à l'égard de la grammaire et de l'orthographe chez les étudiants de magistère de la faculté de pédagogie de Minia (didactique du FLE) en faveur du post/test.

b) Il y a une différence significative entre la moyenne des notes des étudiants du groupe expérimental au pré-post/test de l'écriture académique à l'égard de la syntaxe chez les étudiants de magistère de la faculté de pédagogie de Minia (didactique du FLE) en faveur du post/test.

c) Il y a une différence significative entre la moyenne des notes des étudiants du groupe expérimental au pré-post/test de l'écriture académique à l'égard de la rédaction du texte chez les étudiants de magistère de la faculté de pédagogie de Minia (didactique du FLE) en faveur du post/test.

\section{Outils de la recherche :}

1. Un questionnaire soumis au corps enseignant pour déterminer les erreurs communes de l'écriture académique chez les étudiants de magistère de la faculté de pédagogie de Minia (didactique du FLE) ;

2. Un test diagnostique pour déterminer les erreurs communes de l'écriture académique chez les étudiants de magistère de la faculté de pédagogie de Minia (didactique du FLE). Ce test est, également, utilisé comme pré-post/test en vue de mesurer l'efficacité du programme proposé basé sur l'approche des processus d'écriture pour développer les compétences de l'écriture académique chez les étudiants de magistère de la faculté de pédagogie de Minia (didactique du FLE) ; 
3. Un programme proposé basé sur l'approche des processus d'écriture pour remédier aux erreurs communes de l'écriture académique chez les étudiants de magistère de la faculté de pédagogie de Minia (didactique du FLE). (Outil d'enseignement)

\section{Echantillon de la recherche :}

Cette recherche se délimite à un seul groupe expérimental parmi les étudiants de magistère de la faculté de pédagogie de Minia (didactique du FLE) car ils ont, déjà, étudié les matières qui ont pour objectifs de développer leurs compétences méthodologiques.

\section{Méthodologie de la recherche :}

Afin de réaliser cette recherche, le chercheur utilise une méthode descriptive pour présenter le cadre théorique. Pour vérifier l'efficacité du programme proposé, le chercheur utilise la méthode quasi-expérimentale avec une pré/post évaluation.

\section{Terminologie de l'étude :}

L'écriture académique : L'écriture académique est un type d'écriture utilisé par les chercheurs et les étudiants universitaires dans l'élaboration des rapports de leurs recherches. Ce type d'écriture se caractérise par la précision, la clarté et la brièveté. (Définition opérationnelle)

L'approche des processus d'écriture : L'approche des processus d'écriture est un groupe de processus itératifs qui se déroulent avant (génération des idées - lecture et prise des notes), pendant (faire un brouillon - reformulation) et après l'écriture (évaluation et réécriture). (Définition opérationnelle)

\section{Cadre conceptuel :}

L'écriture est une activité qui joue un rôle très important dans l'élargissement des connaissances et le développement de la réflexion à travers le partage des idées et des informations. Cette activité va d'une tâche simple comme l'écriture d'une lettre à un ami à une tâche complexe comme l'écriture d'un poème. Chaque type d'écriture a ses fonctions et ses caractéristiques.

L'écriture académique est un type d'écriture qui vise la rédaction et le partage des recherches scientifiques et des articles scientifiques universitaires. Cette écriture est adressée à une communauté spécialisée dans un domaine particulier ; elle est, alors, une écriture spécialisée. Bien que l'écriture académique ou l'écriture scientifique ait des caractéristiques générales, l'écriture dans un domaine particulier a ses termes spécifiques à utiliser. Pour une définition plus spécifique, Boch (2013, p. 544) déclare que les écritures académiques sont des « écrits produits par des chercheurs (doctorants ou chercheurs professionnels) ayant pour but la construction et la diffusion du savoir scientifique ».

Dans l'écriture académique, l'auteur doit utiliser une terminologie spécialisée selon son domaine de recherche. La langue de spécialité est distinguée par la présence d'items lexicaux et syntaxiques spécifiques. Il est certes possible de reconnaître les langues de spécialité par la présence d'un vocabulaire particulier, ou par la fréquence d'occurrence d'unités terminologiques (Charnock, 1999). 
Pour les fonctions de l'écriture académique, les chercheurs et les auteurs dans les différents domaines de la science écrivent et font leurs recherches pour plusieurs buts et utilisent l'écriture académique afin d'atteindre ces buts. L'écriture académique a, donc, les mêmes fonctions de la recherche scientifique. Ces fonctions sont déterminées ainsi : (Sydney University, 2019)

- La fonction descriptive : Le type d'écriture académique le plus simple est descriptif. Son but est de fournir des faits ou des informations ;

- La fonction analytique : Il est rare qu'un texte de niveau universitaire soit purement descriptif. La plupart des écrits académiques sont également analytiques. L'écriture analytique inclut l'écriture descriptive, mais on réorganise également les faits et les informations décrites en catégories, groupes, pièces, types ou relations ;

- La fonction persuasive : l'écriture persuasive a toutes les caractéristiques de l'écriture analytique (c'est-à-dire l'information, plus la réorganisation de l'information), avec l'ajout de son propre point de vue. La plupart des essais sont convaincants et il y a au moins un élément de persuasion dans la discussion et la conclusion d'une recherche. Les opinions dans la rédaction académique peuvent inclure un argument, une recommandation, une interprétation des résultats ou une évaluation du travail d'autrui. En écriture persuasive, chaque déclaration doit être étayée par des preuves, comme une référence aux résultats de la recherche ou à des sources publiées.

- La fonction critique : l'écriture critique présente toutes les caractéristiques de l'écriture persuasive, avec un autre point de vue. Bien que l'écriture persuasive exige que le chercheur ait son propre point de vue sur un problème ou un sujet, l'écriture critique requiert qu'il prenne en compte plusieurs points de vue.

Dans une même recherche, le chercheur peut couvrir toutes les fonctions de l'écriture académique tout au long des parties de cette recherche: cadre théorique, méthodologie, analyse et discussion des résultats. Par conséquent, l'écriture académique peut développer chez les étudiants universitaires quelques compétences concernant l'analyse, la critique, la persuasion,... à travers la pratique réelle de ces compétences dans l'écriture.

Pour remplir ces fonctions, chaque article académique ou chaque thèse de recherche doit rédiger à la lumière de quelques principes de la rédaction académique ; ces principes sont déterminés par Whitaker (2009) ainsi : [Traduction libre, p. 2-4]

- Déterminer un but clair :

Le but de l'article est de répondre à la question que le chercheur a posée comme sujet. Cette question lui donne un but. Les objectifs les plus courants dans l'écriture académique sont de persuader, d'analyser / synthétiser et d'informer.

- Utiliser des termes adéquats :

Comme pour toute écriture, l'écriture académique s'adresse à un public spécifique. Le chercheur doit donc utiliser des termes adéquats et suivre un style d'écriture spécifique.

- Présenter ses points de vue : 
La rédaction académique, même dans un but informatif, n'est pas simplement une liste de faits ou des résumés de sources. Bien que le chercheur présente les idées et les recherches d'autres personnes, le but de son document est de montrer ce qu'il pense de ces choses.

- Organiser logiquement le texte :

L'écriture académique suit un schéma organisationnel standard. Pour les essais et articles académiques, il y a une introduction, un corps et une conclusion. Chaque paragraphe mène logiquement au suivant.

- Présenter des explications claires et complètes :

Le lecteur s'attend à ce que tout soit fait pour lui. Il ne devrait pas avoir à réfléchir pour comprendre les idées du chercheur. Les idées doivent, alors, être clairement et complètement expliquées.

- Utiliser des références de qualité :

Le chercheur utilise les références pour soutenir ses propres idées ; par conséquent, le chercheur doit utiliser et compulser des références concernant son sujet. Ces références doivent être professionnelles et académiques de grande qualité ; c'est-à-dire, elles doivent être récentes, d'un nombre suffisant et couvrir toutes les variables de le recherche.

- Suivre correctement le style APA :

Tous les articles académiques dans le domaine de l'éducation doivent suivre les directives de l'Association psychologique américaine concernant les citations dans le texte, la liste de références et le format (il y a plusieurs éditions pour le style APA, on doit suivre la dernière édition : $7^{\text {ème }}$ édition, 2019).

- Suivre un style d'écriture adéquat :

Parce que c'est son travail, le chercheur doit utiliser ses propres mots autant que possible. L'écriture doit être claire, concise et facile à lire. Il est également très important qu'il n'y ait pas d'erreurs de grammaire, d'orthographe, de ponctuation ou de vocabulaire dans les écrits académiques.

En suivant les principes cités ci-dessus, l'auteur de la recherche peut faire une recherche très rigoureuse puisque l'écriture académique s'adresse à un groupe de spécialistes qui doivent arriver à une compréhension fine et exacte des messages adressés par l'auteur. Par conséquent, le style scientifique dans l'écriture académique, contrairement au style de l'écriture littéraire et au style de l'écriture journalistique, doit anticiper et structurer les idées. Il permet au lecteur de comprendre ce qu'il lit. Selon Zabo (2017), le style de l'écriture académique doit être construit autour de quatre principes :

- Le mot est juste, précis, concret pour ne pas avoir d'autres interprétations possibles ;

- La phrase est construite autour d'un verbe d'action et véhicule une seule idée. Elle doit être courte en utilisant des structures grammaticales simples ;

- Le paragraphe commence par la phrase-sujet qui en donne l'idée majeure ;

- Le chercheur explicite et justifie tout ce qu'il écrit, il anticipe en utilisant trois techniques : 
- le début de phrase se réfère au connu déjà évoqué dans le texte, et la fin de phrase amène à l'inconnu (non encore évoqué, nouveau, important) ;

- la répétition des mots et des structure guide le lecteur ;

- les transitions (phrase, expression, mot de liaison) relient les idées, relient le connu à l'inconnu.

Le style de l'écriture académique permet au lecteur de comprendre le sens exact du message adressé par l'auteur puisque un autre lecteur peut comprendre le même sens. Les principes du style de l'écriture académique déterminés ci-dessus nous amènent à déterminer les caractéristiques de l'écriture académique. Maisonneuve (2010) en détermine trois ainsi :

\section{La précision}

Le chercheur doit être rigoureux dans ses observations. La rédaction doit appliquer les principes de rigueur de la démarche scientifique. Il ne doit jamais écrire «environ ..., presque $30 \%$...»; rien n'est précis dans ces structures. On doit déterminer un nombre précis plutôt qu'un pourcentage approximatif. On ne doit pas utiliser les mots « important », " sévère »; car, ces mots peuvent avoir plus d'un sens.

\section{La clarté}

L'idée principale de chaque phrase doit être contenue dans les premiers mots de la phrase. L'idée principale du paragraphe doit être exprimée dans la première phrase du paragraphe. Il s'agit de la position forte. En science, il faut toujours utiliser le même mot pour dire la même chose. L'auteur doit penser à décrire tous les détails pour être clair ; il ne peut pas faire d'ellipses qui obligent les lecteurs à interpréter. Les temps des verbes doivent être utilisés à bon escient : les temps du passé pour les faits passés, les temps du présent pour les faits acquis et les généralisations.

\section{La brièveté}

Les mots qui n'ont pas de message doivent être supprimés. Des mots longs comme « actuellement " ont moins d'information qu'une expression courte comme " en 2020 ». On peut être précis et bref dans la plupart des cas. On ne doit dire les faits qu'une seule fois et ne pas les répéter. Les abréviations ne sont pas toujours un bon moyen pour raccourcir un texte car la lecture est parfois plus difficile. On doit éviter toutes les doubles négations du type « Vous n'utilisez pas de phrases qui n'ont pas de verbes »; il est mieux d'écrire "Toutes les phrases doivent avoir un verbe ». C'est la même idée mais plus facile à comprendre. Plus les mots sont simples et courts, plus les paragraphes sont simples et courts, plus les textes sont lisibles. L'auteur de la recherche doit changer les phrases «négatives » en «positives » (affirmations). Au lieu de dire «Vous devez éviter des mots imprécis », il est mieux d'écrire « Vous utilisez des mots précis».

De plus, L'écriture académique doit se caractériser par l'objectivité qui exige de présenter une discussion équilibrée de différents points de vue. Autrement dit, on doit éviter les jugements de valeur qui impliquent des croyances morales sur ce qui est «juste» ou «faux». Par conséquent, on ne doit pas utiliser des expressions telles que «à mon avis» ou «je pense». De même, on doit éviter l'utilisation de pronoms personnels qui peuvent entraîner des 
biais et l'utilisation des hypothèses non étayées (Smyth, 1996 ; cité par Hartley, 2008) [Traduction libre, p. 3].

Il est probable que la notion d'objectivité soit confuse avec la notion de neutralité. Pour Boch (2013), l'énoncé « neutre » se définit par une absence de prise de point de vue ; au contraire, l'énoncé « objectif » peut comporter des traces du point de vue pris en charge par le locuteur ou l'écrivain. Par conséquent, le développement d'un point de vue, même nuancé, se situe à l'opposé de la neutralité.

La deuxième caractéristique de l'écriture académique relève de sa dimension argumentative où le chercheur conduit une réflexion en développant un point de vue étayé. Un point de vue veut dire que l'auteur choisit un endroit pour étudier son objet et adopte une manière particulière de considérer une question : présenter une problématique, organiser le raisonnement, et sélectionner ses données, justifier ses choix et ses interprétations ; bref, se situer dans l'argumentation scientifique (Boch, 2013).

Le texte d'une thèse de recherche ou d'un article de recherche est semblable à un corps humain dont les parties ou les organes sont en harmonie et en cohérence. Par conséquent, l'écriture académique doit être caractérisée de la cohérence du texte qui concerne l'organisation des représentations configurant l'univers mis en place par le texte. Pour rendre le texte plus cohérent, l'auteur doit utiliser les connecteurs remplissant une fonction de liage sémantique entre l'unité de rangs différents (mots, propositions, portions larges d'un texte) (Paveau et Safarti, 2003 ; Adam, 2005).

Pour commencer à rédiger le rapport de la recherche, l'auteur doit préparer au préalable un certain nombre de fiches servant de base à la rédaction « réservoir d'idées ». Ces fiches doivent être organisées et classées suivant les parties ou les chapitres prévus dans le plan de rédaction. Pour écrire une partie, l'auteur doit commencer par s'imprégner du contenu des fiches correspondantes, puis dégager une idée centrale qui servira de fil directeur au développement. Ensuite, on doit établir un mini plan interne à la partie à écrire, qui détaille l'enchainement des idées suivant les paragraphes, le principe étant : « une idée, un paragraphe » en soignant les transitions entre les parties, l'enchainement logique des idées et le cheminement argumentatif des paragraphes (Guidère, 2004).

Les compétences de l'écriture académique doivent être enseignées et développées chez les jeunes chercheurs. Dans le contexte de l'enseignement de l'écriture, il est utile d'examiner trois approches influentes dans ce domaine: écrire en tant que texte, écrire en tant que processus et écrire en tant que pratique sociale (Coffin et al, 2005 [Traduction libre, p. 910]) :

- Approche textuelle : dans cette approche, l'accent est mis sur l'écriture des étudiants en tant que textes finaux ou «produits». Cette approche exige de présenter aux étudiants des «modèles de bonne écriture», et leur demander d'imiter ces modèles. L'accent est mis, alors, sur les caractéristiques spécifiques des textes écrits, par exemple l'orthographe, la structure du texte, le vocabulaire et le style. De plus, les processus d'écriture, y compris les décisions conscientes et inconscientes prises par les étudiants écrivains, n'est généralement pas pris en considération. 
- Approche des processus: L'approche des processus d'écriture met l'accent sur le traitement des étapes de l'écriture que l'apprenant peut suivre (On va traiter cette approche en détail dans les lignes suivantes).

- L'écriture en tant que pratique sociale : L'accent est mis ici sur l'écriture en tant qu'activité se déroulant toujours dans un contexte social. Dans le contexte de l'enseignement supérieur, l'écriture des étudiants peut être comprise comme une «pratique sociale» car l'écriture des étudiants est ancrée dans les relations autour de l'enseignement et de l'apprentissage. De même, les conventions des normes de l'écriture académique se sont développées au sein de communautés académiques et disciplinaires spécifiques. De plus, l'écriture académique est une pratique sociale puisque les étudiants apprennent non seulement à communiquer de manière particulière, mais aussi à apprendre à être spécialistes dans des sciences sociales. Ainsi, l'écriture académique est une identité personnelle et sociale.

L'approche des processus d'écriture est un groupe d'activités variées qui contribuent à l'apprentissage de l'écriture à travers des activités interactives telles que le remue-méninges, les discussions en groupe, la réécriture ... Dans cette approche, les besoins, les attentes, les objectifs, les styles d'apprentissage, les compétences et les connaissances des apprenants sont pris en compte. Tout au long des processus de rédaction, les apprenants doivent tirer les meilleures de leurs capacités telles que les connaissances et les compétences, en profitant de l'aide et de la coopération appropriées du formateur et des autres apprenants. Cette approche encourage les apprenants à se sentir libres d'exprimer leurs idées ou leurs sentiments dans des massages écrits en leur laissant suffisamment de temps et d'occasion de réviser leur écriture et de faire appel à des ressources extérieures telles que le formateur (Onozawa, 2010 [traduction libre, p. 155]).

L'approche des processus d'écriture peut être utilisée d'une manière simple ou complexe ; c'est-à-dire que les chercheurs peuvent adopter une approche ayant trois phases : la phase de la pré-écriture, la phase de l'écriture et la phase de la révision. D'autres chercheurs adoptent une approche ayant cinq phases ou plus : la phase de pré-écriture, la phase de rédaction d'un brouillon, la phase de la révision, la phase de la réécriture, la phase de l'évaluation et la phase de la publication.

Les étapes de l'approche des processus d'écriture sont généralement mises en œuvre de manière séquentielle, mais dans le processus d'écriture, il n'est pas nécessairement un processus d'apprentissage linéaire, c'est plutôt un processus récursif ou en spirale au fur et à mesure que les apprenants avancent dans ces étapes, allant parfois jusqu'à revenir en arrière. Par exemple, le remue-méninges, une stratégie importante en particulier pour l'étape de préécriture, peuvent être exercés à différentes étapes si l'apprenant a besoin de nouvelles idées plus tard dans les processus d'écriture. (Onozawa, 2010) [Traduction libre, p. 155].

Pour les caractéristiques de l'approche des processus d'écriture, elles sont décrites par Hairston (1988 ; cité par Ouskourt, 2008) ainsi : [traduction libre, p. 70-71]

- Le formateur intervient auprès les étudiants pendant les processus d'écriture.

- Le formateur aide les étudiants à déterminer les objectifs et à générer du contenu. 
- Le formateur évalue le produit écrit en fonction de la capacité de l'étudiant à répondre à l'intention de l'auteur et aux besoins du public.

- On considère l'écriture comme un processus récursif plutôt que linéaire.

- On souligne que l'écriture est un moyen d'apprendre et de se développer, ainsi qu'une compétence communicative.

- On inclut une variété de modes d'écriture expressifs et explicatifs.

- Cette approche s'appuie sur d'autres disciplines, notamment la psychologie cognitive et la linguistique.

- On considère l'écriture comme une activité créative disciplinée pouvant être analysée et décrite.

- Cette approche est basée sur la recherche linguistique et sur les processus de composition.

- On souligne le principe selon lequel le formateur doit être une personne qui pratique l'écriture.

Selon ces caractéristiques, le formateur est considéré comme facilitateur ; il dirige l'apprenant vers la détermination du sujet de l'écriture, la création des idées, la prise des notes (dans le cas d'utiliser des références), l'écriture d'un brouillon, l'autoévaluation, l'évaluation par les pairs. Le formateur fait, également une évaluation formative et sommative.

L'approche des processus repose sur la notion selon laquelle l'écriture est un processus qualificatif. Les étapes des processus d'écriture peuvent se dérouler dans différents ordres et à différents moments. Ces étapes sont déterminées par Coffin et al, (2005) ainsi : [traduction libre, p. 34-42]

La phase de la pré-écriture : Dans cette phase, l'étudiant-écrivain utilise des stratégies de rédaction telles que le remue-méninges, la prise de notes, la lecture/relecture, la lecture/tutoriel et l'écriture libre qui l'aident à trouver des idées, à comprendre les idées des autres, à collecter des informations, à activer les connaissances antérieures et à organiser leurs idées. Les étudiants trouvent souvent utile de réfléchir à des idées avant d'essayer de créer un texte. Certaines de ces stratégies peuvent être bien utilisées individuellement; d'autres peuvent être mieux exécutées en binôme ou en petit groupe.

L'écriture d'un brouillon : L'un des principes fondamentaux de l'approche de processus est que l'écriture est un processus itératif. Ainsi, dans la mesure du possible, les tâches écrites devraient s'appuyer sur la révision de l'écriture en réponse aux commentaires des pairs examinateurs ou du formateur. Les étudiants sont plus susceptibles de profiter des commentaires du formateur sur leur écriture s'ils auront l'occasion de le retravailler. De plus, les étudiants qui écrivent et redéfinissent leur travail ont moins l'occasion de présenter un travail plagié. Dans une première version, l'étudiant devrait se concentrer sur le développement du sens en utilisant les idées rassemblées dans les stratégies de pré-écriture.

L'évaluation par les pairs : La révision par les pairs est l'un des aspects les plus importants des processus d'écriture. Les commentaires sur les projets des étudiants peuvent prendre la 
forme de commentaires oraux ou écrits des pairs ou du formateur pour guider les étudiants dans leur travail. Avec leur formation et leur pratique, les étudiants peuvent, également, développer leurs compétences critiques. Après l'évaluation par les pairs, l'étudiant auteur peut poser des questions de clarification sur les commentaires présentés. Enfin, l'étudiant écrivain devrait essayer de résumer une stratégie pour répondre aux commentaires des examinateurs dans les révisions ultérieures.

La réflexion : Une phase de réflexion donnée aux étudiants écrivains pour leur permettre de voir les lacunes dans leur structure de texte, l'utilisation de preuves, etc.

L'édition : Les dernières étapes de l'écriture consistent en l'édition, la correction des erreurs et le polissage d'un texte. Tous les étudiants peuvent bénéficier des informations et des exemples d'utilisation donnés dans les dictionnaires et les ouvrages de référence écrits. Il est de plus en plus demandé aux étudiants de s'engager dans la rédaction collaborative. Lorsqu'il s'agit de rédiger des textes en collaboration, les étudiants peuvent être responsables de la rédaction de différentes sections du texte.

Pour suivre l'approche des processus d'écriture, les auteurs ont créé plusieurs modèles : le modèle de Hayes et Flower (1980), le modèle de White et Arndt (1991), le modèle de révision de Hayes (1996), le modèle de Hayes (2012), le modèle d'Abas et Abd Aziz (2016). De même, le chercheur présente son propre modèle à suivre dans la présente étude.

\section{Le modèle de Hayes et Flower (1980) :}

Le modèle de Hayes et Flower (1980) (figure 1) implique 3 phases des processus d'écriture : la planification, la mise en texte et la révision. Dans la planification, l'étudiant écrivain planifie son écriture en cherchant des idées (génération d'idées) qu'il les trie et les structure (organisation) en fonction des buts qu'il s'est fixés (cadrage des idées). La mise en texte est l'opération qui consiste à écrire le texte, à mettre en mots, en phrases et en texte les idées retenues. Lors de la révision, l'étudiant écrivain lit, évalue et modifie son texte, si nécessaire, pour s'assurer qu'il soit cohérent et sans erreur. Ce modèle présente les processus d'écriture comme des processus non linéaires ; l'étudiant écrivain peut faire des allers retours entre la planification, la mise en texte et la révision. Dans ce modèle, la mémoire à long terme de l'étudiant écrivain joue un rôle important puisqu'il y puise des connaissances sur le sujet qu'il doit écrire. De même, le sujet de l'écriture doit être présenté dans un contexte (un problème rencontré lors de l'écriture et le texte qu'on a déjà écrit). 


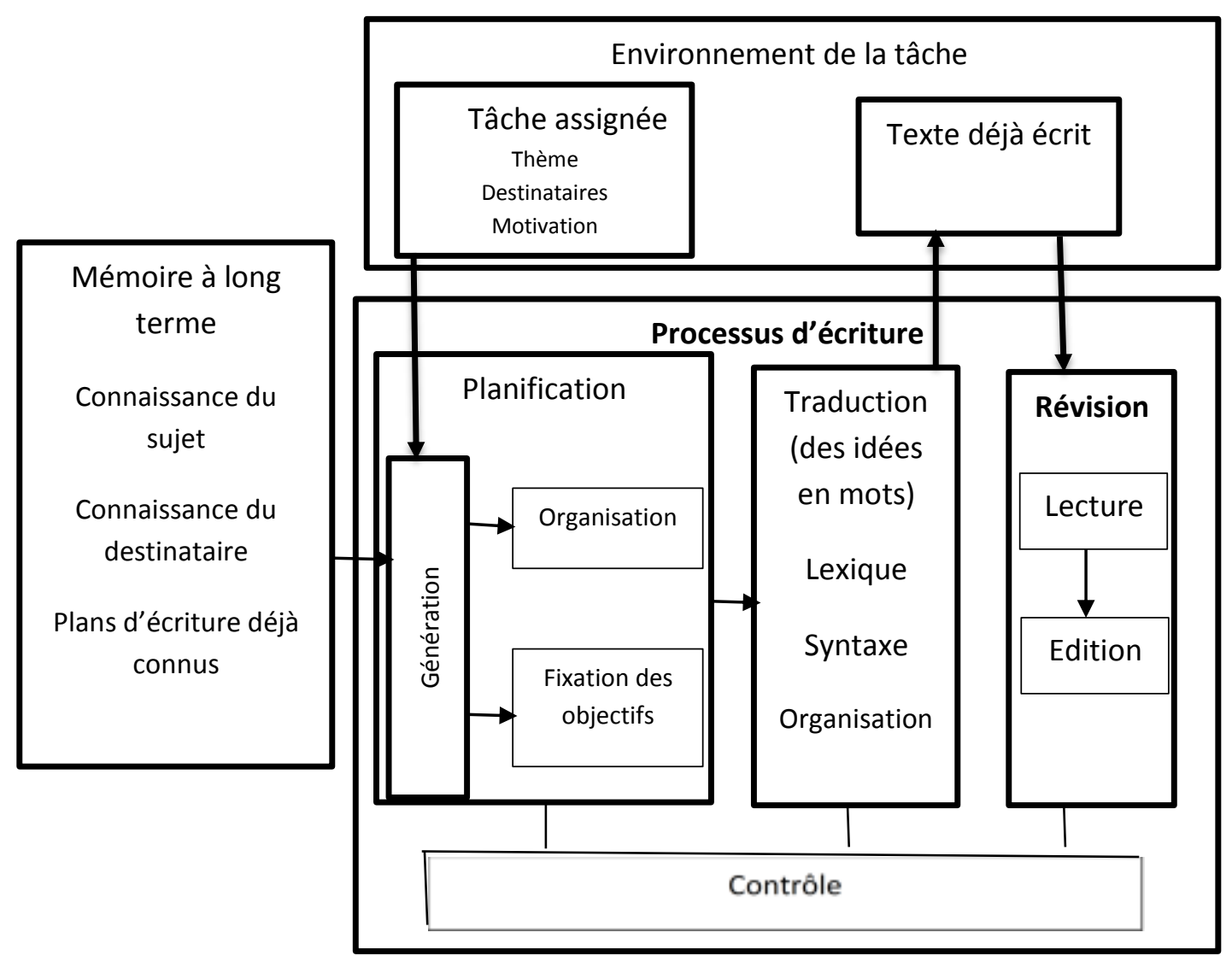

Figure ' . Modèle des processus d'écriture adapté de Hayes et Flower (1980, p. 11)

\section{Le modèle de White et Arndt (1991) :}

Selon White et Arndt (1991), les processus d'écriture se représentent dans un cycle de génération d'idées, de structuration, d'écrire un brouillon, de révision, de mise au point et d'évaluation (figure 2).

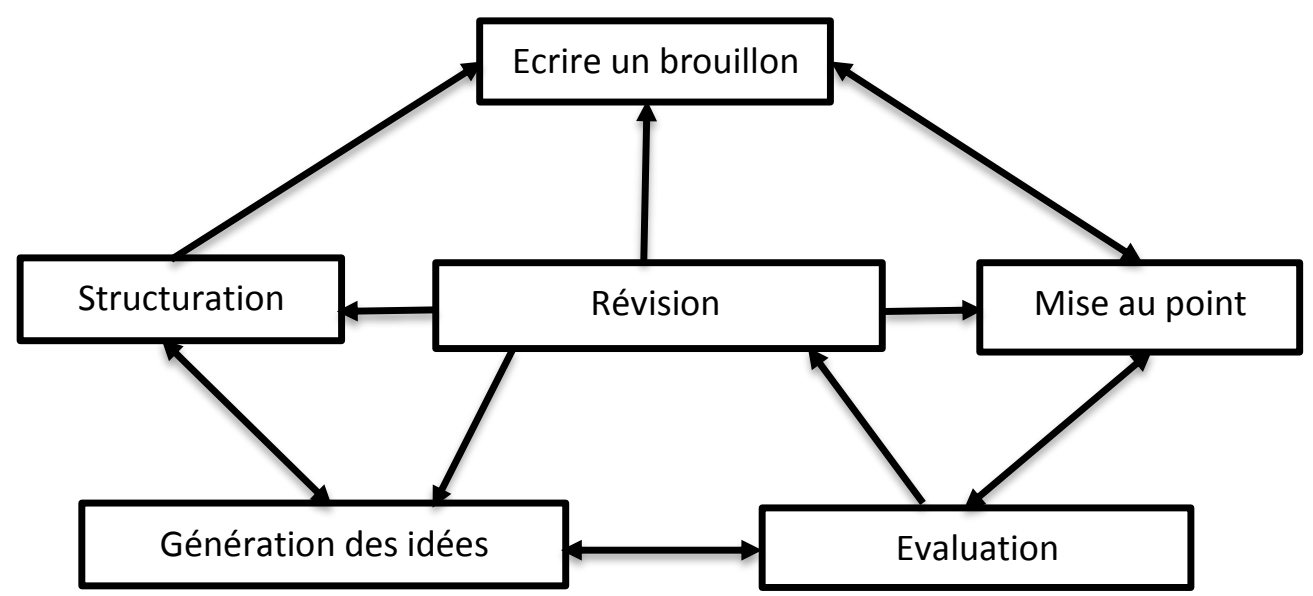

Figurer .Modèle des processus d'écriture (White and Arndt, 1991:43)

Cette figure montre que l'écriture passe par des processus non linéaire ; elle implique un cycle de réflexion (génération des idées), d'écriture et de révision. 


\section{Le modèle de révision de Hayes (1996)}

Dans le modèle de révision de Hayes (figure 3), les processus fondamentaux d'écriture sont la réflexion, la production du texte, (la résolution de problèmes - la prise de décisions) et le traitement du texte (la lecture critique). De même, la mémoire à long terme et la mémoire de travail sont considérées comme des ressources pour l'écriture.

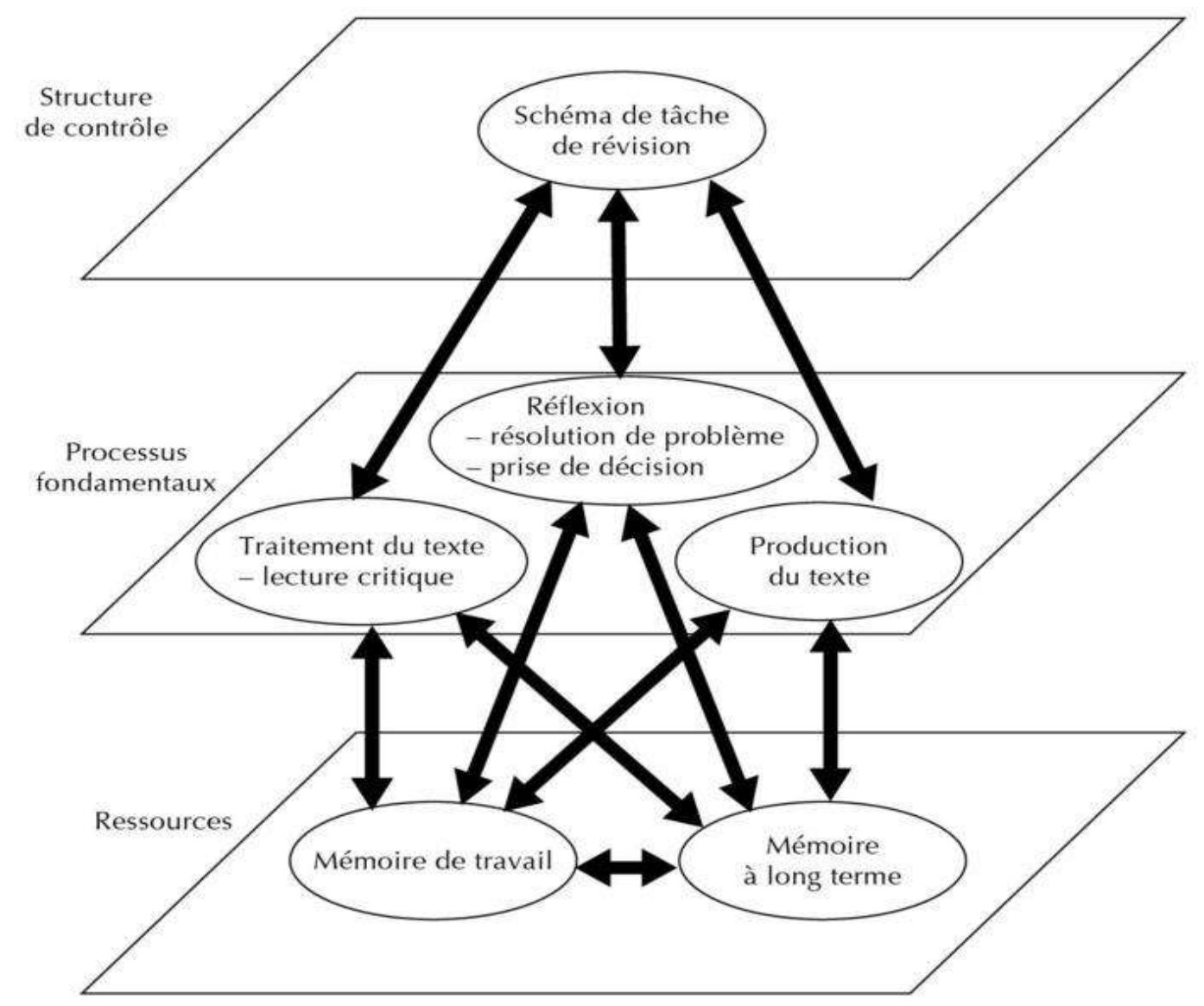

Figure 3. Modèle des processus d'écriture (Hayes, 1996:17)

\section{Le modèle modifié de Hayes (2012) :}

Selon le modèle modifié de Hayes (figure 4), les processus d'écriture sont divisés en trois niveaux :

- Niveau des ressources : ce niveau est la capacité à mobiliser les connaissances antérieures pour rendre les processus cognitifs de l'écriture plus efficaces.

- Niveau des processus: ce niveau inclut les habiletés cognitives, ainsi que l'environnement de la tâche. Dans ce niveau, les moyens technologiques peuvent compenser pour un processus cognitif déficitaire.

- Niveau du contrôle : ce niveau représente l'aspect métacognitif (la capacité à prendre conscience de son propre fonctionnement cognitif et la capacité d'autoréguler ses stratégies). 


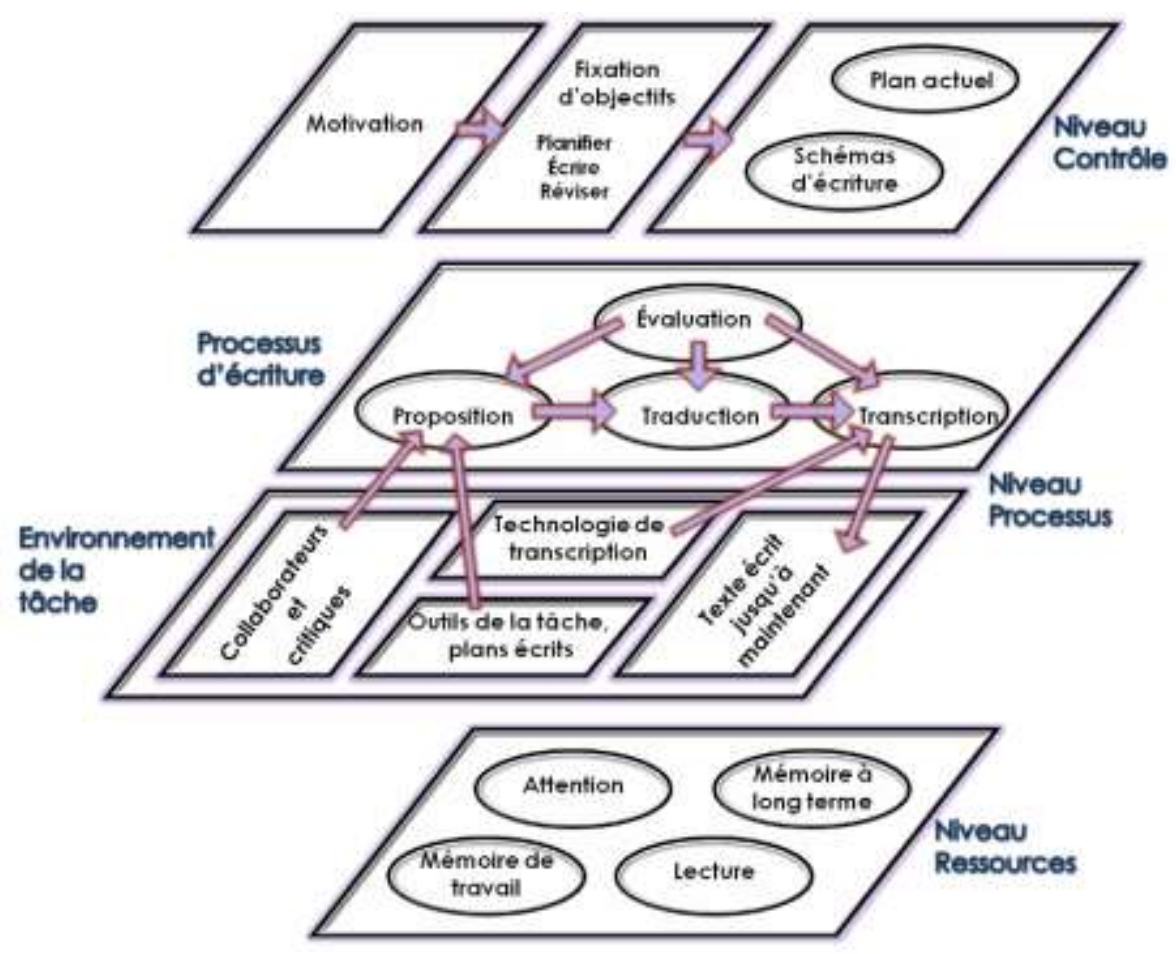

Figure 4. Modèle des processus d'écriture (Hayes, 2012, p. 371)

\section{Le modèle d'Abas et Abd Aziz (2016) :}

Abas et Abd Aziz (2016) a mené une étude pour déterminer le modèle selon lequel les apprenants suivent les processus d'écriture. Dans la tâche d'écriture, les participants commencent avec l'étape de la pré-écriture, en utilisant diverses stratégies telles que la mise en plan, la liste, la discussion écrite et l'écriture libre. Ensuite, les participants poursuivent une phase de planification au cours de laquelle des stratégies telles que la prise en compte du lecteur sont prises en considération. Dans la phase de rédaction, les participants commencent à rédiger leur contenu en paragraphes. À ce stade, les participants peuvent s'arrêter pour lire ce qu'ils écrivent, s'assurer qu'ils suivent leur plan et leurs notes de pré-écriture. Les apprenants écrivains relient le sujet à leur expérience passée. Lorsque les participants sont à court d'idées, ils font une pause et ils lisent. À ce stade, des stratégies telles que la recherche d'aide et l'utilisation de matériel en ligne sont mises en place. La révision et l'édition ont lieu simultanément, au moment silencieux où les participants s'arrêtent et lisent ce qu'ils ont écrit (Abas and Abd Aziz, 2016, p. 377). 


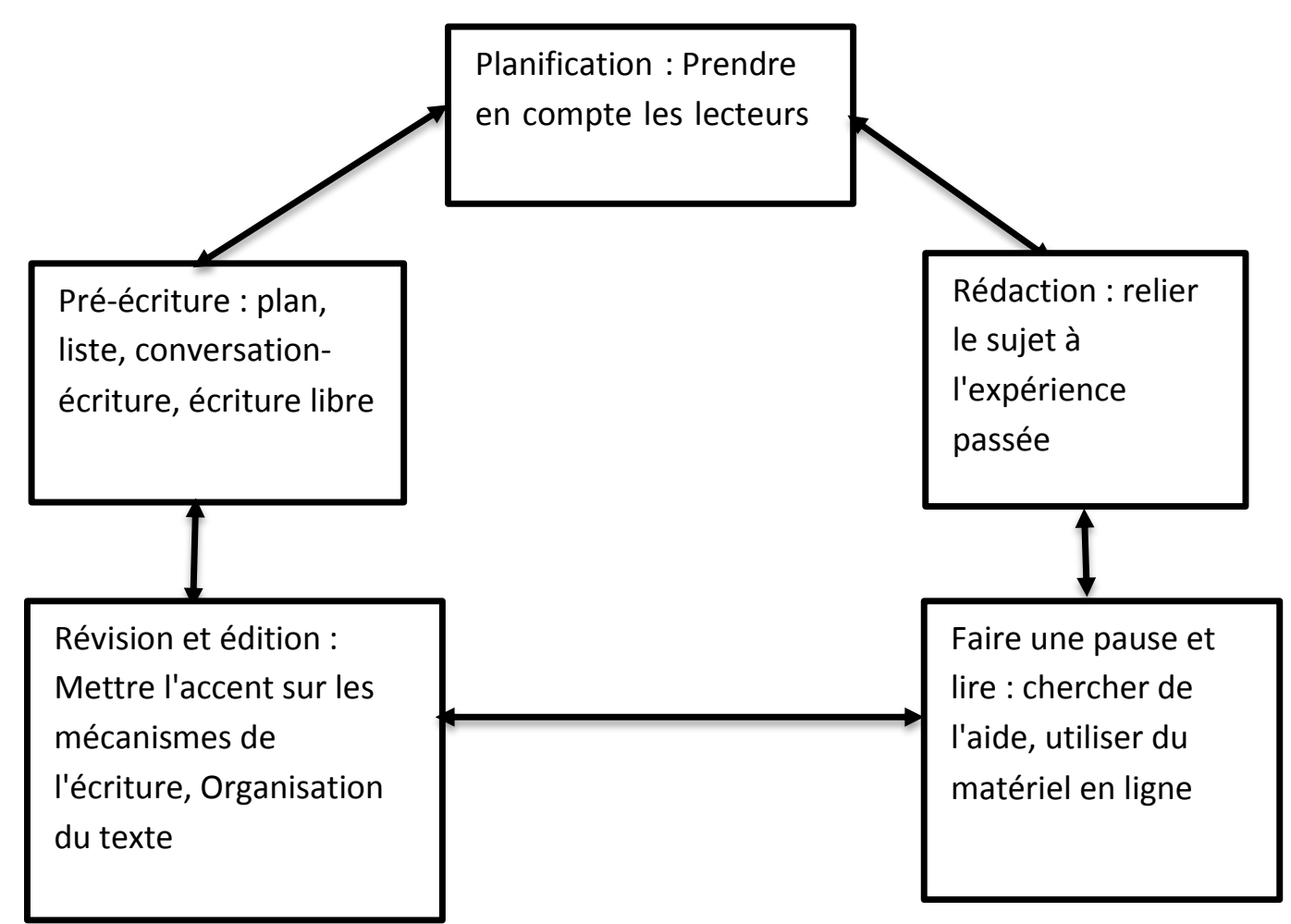

Figure 5. Modèle des processus d'écriture (Abas \& Abd Aziz, 2016, p. 377)

Dans notre étude, nous proposons le modèle suivant pour la stratégie des processus d'écriture (figure 6) :

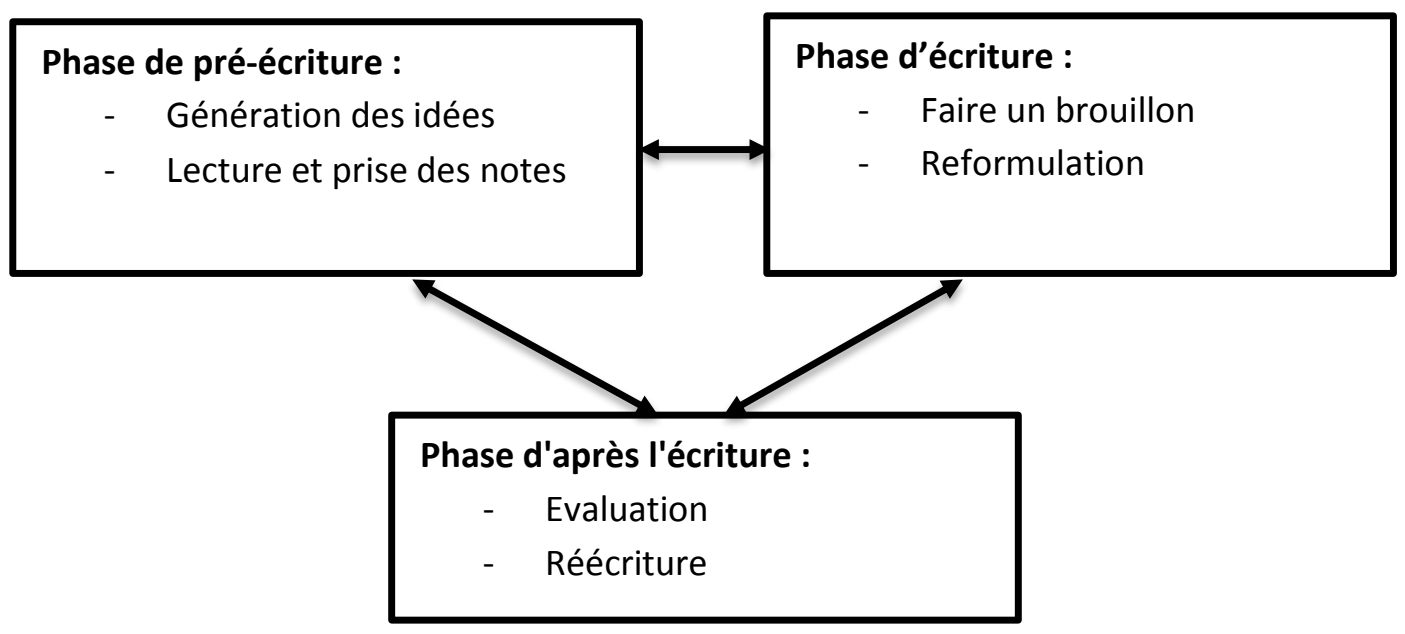

Figure 5. Modèle des processus d'écriture élaboré par le chercheur)

Notre modèle des processus d'écriture se compose de 3 phases : phase de pré-écriture (Génération des idées - Lecture et prise des notes), phase d'écriture (Faire un brouillon Reformulation) et phase d'après l'écriture (Evaluation - Réécriture). Dans la phase de préécriture, les étudiants écrivains passent une séance de remue-méninges pour générer les idées principales et les idées secondaires du texte. Après avoir déterminé les idées, ils compulsent la littérature et les études antérieures et prennent des notes lors de leur lecture. Dans la phase de 
l'écriture, les étudiants écrivains font un brouillon pour le texte. Ensuite, ils reformulent ce qu'ils ont écrit avec leur propre style. Dans la phase d'après l'écriture, les écrits sont évalués, soit par une autoévaluation, soit par une évaluation par les pairs, soit par une évaluation par le formateur. Dans cette phase, les étudiants écrivains réécrivent leur texte à la lumière des évaluations passées. Ce modèle insiste sur le fait que l'écriture est un processus récursif ; c'est-à-dire, on peut revenir sur une phase précédente en cas de besoin.

\section{Matériels et méthodes}

\section{Elaboration d'un questionnaire pour déterminer les erreurs communes en écriture académique :}

Pour collecter les données afin de vérifier l'efficacité du programme basé sur les processus d'écriture pour remédier aux erreurs communes de l'écriture académique chez les étudiants de magistère en didactique du FLE, un questionnaire a été élaboré pour déterminer les erreurs communes de l'écriture académique aux étudiants de magistère à la faculté de pédagogie (didactique du FLE).

Pour élaborer le questionnaire de type Likert, une sélection des items a été faite à la lumière du cadre théorique de cette recherche. Cette première sélection a été complétée par la rédaction de nouveaux items à la lumière de la littérature et des études antérieures. Pour s'assurer de la clarté des items, le chercheur a discuté avec deux experts en didactique du FLE. Une modification sur la forme et le fond de certains items et une élimination des items redondants ont été faites à la lumière de cette discussion. Notre questionnaire se composait de 82 items ; après avoir exécuté la modification nécessaire, le questionnaire devient de 78 items. Pour chaque item, le répondant a trois possibilités de choix (oui $=5$ points, parfois $=3$ points et non $=1$ point $)$.

\section{Validation du questionnaire :}

Le chercheur a traité les données avec le logiciel Statistique SPSS (version 23 Windows). Ce traitement des données a permis de faire une analyse descriptive des résultats. La validité de construit a été vérifiée par la méthode de l'analyse factorielle exploratoire. Les corrélations des items- total varient entre 0.38 et 0.72 .

Quant à la cohérence interne, elle a été évaluée par le calcul du coefficient alpha de Cronbach. L'indice de consistance de l'échelle est constitué quand l'alpha de Cronbach varie entre 0 et 1 . La consistance interne de notre instrument est grande (0.89).

Administration du questionnaire :

Le questionnaire a été soumis auprès des membres du corps enseignants (10) aux facultés de pédagogie pendant le deuxième semestre de l'année universitaire 2018/2019.

Résultats du questionnaire :

Après avoir administré le questionnaire auprès des membres du corps enseignant aux facultés de pédagogie (10). Nous avons donné aux choix des réponses les notes suivantes : 
Non $=$ un pont, Parfois $=3$ points et Oui $=5$ points. Nous présentons les résultats du questionnaire ainsi :

\begin{tabular}{|c|c|c|c|c|c|c|}
\hline \multirow[b]{2}{*}{$\mathbf{N}^{\circ}$} & \multirow[b]{2}{*}{ Items } & \multicolumn{3}{|c|}{ Pourcentage } & \multirow{2}{*}{ 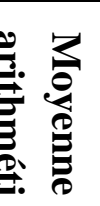 } & \multirow{2}{*}{ 党 } \\
\hline & & 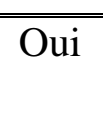 & $\begin{array}{c}\text { Parf } \\
\text { ois }\end{array}$ & Non & & \\
\hline 6 & $\begin{array}{l}\text { Le titre de la recherche est très long } \\
\text { (plus de } 15 \text { mots) ; }\end{array}$ & $80 \%$ & $20 \%$ & $0 \%$ & 4.6 & .814 \\
\hline 29 & $\begin{array}{l}\text { Erreurs dans la reformulation en cas } \\
\text { de la citation indirecte ; }\end{array}$ & $80 \%$ & $20 \%$ & $0 \%$ & 4.6 & .814 \\
\hline 41 & $\begin{array}{lll}\begin{array}{l}\text { Erreurs dans l'utilisation } \\
\text { connecteurs ; }\end{array} & \text { des } \\
\end{array}$ & $80 \%$ & $20 \%$ & $0 \%$ & 4.6 & .814 \\
\hline 22 & $\begin{array}{l}\text { Compter, dans le cadre théorique, sur } \\
\text { les citations et ne pas présenter ses } \\
\text { propres idées ; }\end{array}$ & $70 \%$ & $30 \%$ & $0 \%$ & 4.4 & .932 \\
\hline 23 & $\begin{array}{l}\text { Ne pas respecter le principe «Une } \\
\text { idée pour chaque paragraphe »; }\end{array}$ & $70 \%$ & $30 \%$ & $0 \%$ & 4.4 & .932 \\
\hline 40 & $\begin{array}{l}\text { Erreurs dans l'utilisation de la } \\
\text { ponctuation ; }\end{array}$ & $70 \%$ & $30 \%$ & $0 \%$ & 4.4 & .932 \\
\hline 11 & $\begin{array}{l}\text { L'introduction ne présente pas le } \\
\text { contexte (l'apport de la recherche } \\
\text { dans le domaine par rapport aux } \\
\text { études antérieures) dans lequel } \\
\text { s'inscrit la recherche; }\end{array}$ & $60 \%$ & $40 \%$ & $0 \%$ & 4.2 & .997 \\
\hline 46 & $\begin{array}{l}\text { Les idées présentées manquent de } \\
\text { l'argumentation ; }\end{array}$ & $60 \%$ & $40 \%$ & $0 \%$ & 4.2 & .997 \\
\hline 68 & $\begin{array}{l}\text { Ne pas rédiger une conclusion } \\
\text { convenable pour la recherche ; }\end{array}$ & $70 \%$ & $20 \%$ & $10 \%$ & 4.2 & $\begin{array}{c}1.34 \\
9\end{array}$ \\
\hline 69 & $\begin{array}{l}\text { Ne pas présenter les limites de la } \\
\text { recherche; }\end{array}$ & $70 \%$ & $20 \%$ & $10 \%$ & 4.2 & $\begin{array}{c}1.34 \\
9\end{array}$ \\
\hline 24 & Rédiger des paragraphes très courts ; & $60 \%$ & $30 \%$ & $10 \%$ & 4.0 & $\begin{array}{c}1.36 \\
5\end{array}$ \\
\hline 66 & $\begin{array}{l}\text { Interpréter les résultats de la } \\
\text { recherche sans tenir compte le cadre } \\
\text { théorique ; }\end{array}$ & $60 \%$ & $30 \%$ & $10 \%$ & 4.0 & $\begin{array}{c}1.36 \\
5\end{array}$ \\
\hline 67 & $\begin{array}{l}\text { Manque dans la discussion des } \\
\text { résultats; }\end{array}$ & $60 \%$ & $30 \%$ & $10 \%$ & 4.0 & 1.365 \\
\hline 27 & $\begin{array}{l}\text { Ne pas rédiger une conclusion } \\
\text { convenable pour chaque axe de la } \\
\text { recherche; }\end{array}$ & $60 \%$ & $20 \%$ & $20 \%$ & 3.8 & 1.627 \\
\hline 30 & $\begin{array}{l}\text { Erreurs dans l'utilisation du lexique } \\
\text { et des expressions ; }\end{array}$ & $50 \%$ & $40 \%$ & $10 \%$ & 3.8 & 1.349 \\
\hline
\end{tabular}




\begin{tabular}{|c|c|c|c|c|c|c|}
\hline \multirow[b]{2}{*}{$\mathbf{N}^{\circ}$} & \multirow[b]{2}{*}{ Items } & \multicolumn{3}{|c|}{ Pourcentage } & \multirow{2}{*}{ 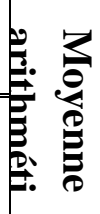 } & \multirow{2}{*}{ 胥 } \\
\hline & & Oui & $\begin{array}{l}\text { Parf } \\
\text { ois }\end{array}$ & Non & & \\
\hline 61 & $\begin{array}{l}\text { Ne pas décrire suffisamment les outils } \\
\text { de collecte de données ; }\end{array}$ & $60 \%$ & $20 \%$ & $20 \%$ & 3.8 & $\begin{array}{c}1.62 \\
7\end{array}$ \\
\hline 64 & $\begin{array}{l}\text { Interpréter insuffisamment les } \\
\text { résultats de la recherche ; }\end{array}$ & $60 \%$ & $20 \%$ & $20 \%$ & 3.8 & $\begin{array}{c}1.62 \\
7\end{array}$ \\
\hline 65 & $\begin{array}{l}\text { Interpréter les résultats de la } \\
\text { recherche sans tenir compte les } \\
\text { résultats des études antérieures ; }\end{array}$ & $60 \%$ & $20 \%$ & $20 \%$ & 3.8 & $\begin{array}{c}1.62 \\
7\end{array}$ \\
\hline 74 & $\begin{array}{l}\text { Compter sur beaucoup de ressources } \\
\text { anciennes ; }\end{array}$ & $50 \%$ & $40 \%$ & $10 \%$ & 3.8 & $\begin{array}{c}1.34 \\
9\end{array}$ \\
\hline 75 & $\begin{array}{l}\text { Ne pas suivre une méthode } \\
\text { convenable }(\mathrm{APA}) \text { dans la rédaction } \\
\text { des références ; }\end{array}$ & $50 \%$ & $40 \%$ & $10 \%$ & 3.8 & $\begin{array}{c}1.34 \\
9\end{array}$ \\
\hline 8 & $\begin{array}{l}\text { Ne pas faire un équilibre entre les } \\
\text { éléments du résumé ; }\end{array}$ & $50 \%$ & $30 \%$ & $20 \%$ & 3.6 & 1.589 \\
\hline 20 & $\begin{array}{l}\text { Ne pas citer les raisons de la } \\
\text { délimitation de la recherche ; }\end{array}$ & $50 \%$ & $30 \%$ & $20 \%$ & 3.6 & 1.589 \\
\hline 43 & Ecrire des phrases très longues ; & $50 \%$ & $30 \%$ & $20 \%$ & 3.6 & 1.589 \\
\hline 47 & Le texte manque de la cohérence ; & $50 \%$ & $30 \%$ & $20 \%$ & 3.6 & 1.589 \\
\hline 48 & $\begin{array}{l}\text { Traduire les idées littéralement de } \\
\text { l'arabe en français ; }\end{array}$ & $50 \%$ & $30 \%$ & $20 \%$ & 3.6 & 1.589 \\
\hline 60 & $\begin{array}{l}\text { Utiliser des moyens inadéquats pour } \\
\text { mesurer la validité et la stabilité des } \\
\text { outils de collecte de données ; }\end{array}$ & $50 \%$ & $30 \%$ & $20 \%$ & 3.6 & 1.589 \\
\hline 73 & $\begin{array}{l}\text { Compter, essentiellement, sur des } \\
\text { ressources secondaires ; }\end{array}$ & $50 \%$ & $30 \%$ & $20 \%$ & 3.6 & 1.589 \\
\hline 32 & $\begin{array}{l}\text { Utiliser beaucoup de sous-titres qui } \\
\text { peuvent perturber l'homogénéité du } \\
\text { texte; }\end{array}$ & $50 \%$ & $20 \%$ & $30 \%$ & 3.4 & $\begin{array}{c}1.77 \\
3\end{array}$ \\
\hline 34 & Ne pas utiliser le temps convenable ; & $50 \%$ & $20 \%$ & $30 \%$ & 3.4 & $\begin{array}{c}1.77 \\
3\end{array}$ \\
\hline 35 & $\begin{array}{l}\text { Erreurs dans la conjugaison des } \\
\text { verbes; }\end{array}$ & $50 \%$ & $20 \%$ & $30 \%$ & 3.4 & $\begin{array}{c}1.77 \\
3\end{array}$ \\
\hline 44 & $\begin{array}{l}\text { Les idées dans le texte ne sont pas } \\
\text { enchainées ; }\end{array}$ & $40 \%$ & $40 \%$ & $20 \%$ & 3.4 & 1.522 \\
\hline 63 & $\begin{array}{l}\text { Ne pas utiliser les tableaux et les } \\
\text { figures nécessaires pour présenter les } \\
\text { résultats de la recherche ; }\end{array}$ & $50 \%$ & $20 \%$ & $30 \%$ & 3.4 & $\begin{array}{c}1.77 \\
3\end{array}$ \\
\hline 78 & Ne pas faire un équilibre entre les & $50 \%$ & $20 \%$ & $30 \%$ & 3.4 & 1.77 \\
\hline
\end{tabular}




\begin{tabular}{|c|c|c|c|c|c|c|}
\hline \multirow[b]{2}{*}{$\mathbf{N}^{\circ}$} & \multirow[b]{2}{*}{ Items } & \multicolumn{3}{|c|}{ Pourcentage } & \multirow{2}{*}{ 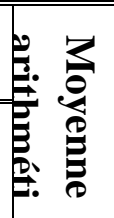 } & \multirow{2}{*}{ 常 } \\
\hline & & Oui & $\begin{array}{c}\text { Parf } \\
\text { ois }\end{array}$ & Non & & \\
\hline & parties de la recherche ; & & & & & 3 \\
\hline 9 & $\begin{array}{l}\text { L'introduction n'a pas de } \\
\text { développement logique qui part du } \\
\text { plus général vers le plus spécifique ; }\end{array}$ & $40 \%$ & $30 \%$ & $30 \%$ & 3.2 & 1.690 \\
\hline 12 & $\begin{array}{l}\text { L'introduction ne conduit pas à la } \\
\text { détermination et à la formulation de } \\
\text { la problématique de la recherche ; }\end{array}$ & $40 \%$ & $30 \%$ & $30 \%$ & 3.2 & 1.690 \\
\hline 16 & $\begin{array}{l}\text { la problématique est déterminée sous } \\
\text { forme d'une question ; }\end{array}$ & $40 \%$ & $30 \%$ & $30 \%$ & 3.2 & 1.690 \\
\hline 19 & $\begin{array}{l}\text { Erreurs dans la rédaction des } \\
\text { objectifs de la recherche ; }\end{array}$ & $40 \%$ & $30 \%$ & $30 \%$ & 3.2 & 1.690 \\
\hline 52 & $\begin{array}{l}\text { Ne pas citer les études antérieures } \\
\text { dans le contexte convenable; }\end{array}$ & $40 \%$ & $30 \%$ & $30 \%$ & 3.2 & 1.690 \\
\hline 56 & $\begin{array}{l}\text { Ne pas utiliser une manière adéquate } \\
\text { dans le choix de l'échantillon ; }\end{array}$ & $40 \%$ & $30 \%$ & $30 \%$ & 3.2 & 1.690 \\
\hline 70 & $\begin{array}{l}\text { Présenter des recommandations } \\
\text { inappropriées ; }\end{array}$ & $40 \%$ & $30 \%$ & $30 \%$ & 3.2 & 1.690 \\
\hline 7 & $\begin{array}{l}\text { Le résumé manque d'informations } \\
\text { essentielles ; }\end{array}$ & $40 \%$ & $20 \%$ & $40 \%$ & 3.0 & 1.819 \\
\hline 10 & $\begin{array}{l}\text { L'introduction n'éclaircit pas } \\
\text { l'importance de la recherche ; }\end{array}$ & $30 \%$ & $40 \%$ & $30 \%$ & 3.0 & 1.576 \\
\hline 31 & $\begin{array}{l}\text { Utiliser des termes inadéquats pour le } \\
\text { domaine ; }\end{array}$ & $30 \%$ & $40 \%$ & $30 \%$ & 3.0 & 1.576 \\
\hline 39 & $\begin{array}{l}\text { Erreurs dans les accords en genre et } \\
\text { en nombre ; }\end{array}$ & $30 \%$ & $40 \%$ & $30 \%$ & 3.0 & 1.576 \\
\hline 42 & $\begin{array}{l}\text { Erreurs dans la construction de la } \\
\text { phrase (ordre des mots dans la } \\
\text { phrase); }\end{array}$ & $30 \%$ & $40 \%$ & $30 \%$ & 3.0 & 1.576 \\
\hline 49 & $\begin{array}{l}\text { La revue de littérature ne montre pas } \\
\text { l'évolution d'un courant de pensée, } \\
\text { d'une théorie, d'une méthode, etc. }\end{array}$ & $30 \%$ & $40 \%$ & $30 \%$ & 3.0 & 1.576 \\
\hline 59 & $\begin{array}{l}\text { Erreurs dans l'élaboration des outils } \\
\text { de collecte de données ; }\end{array}$ & $30 \%$ & $40 \%$ & $30 \%$ & 3.0 & 1.576 \\
\hline 72 & $\begin{array}{l}\text { Erreurs dans la rédaction des } \\
\text { références (auteur, date, lieu de la } \\
\text { publication,...); }\end{array}$ & $30 \%$ & $40 \%$ & $30 \%$ & 3.0 & 1.576 \\
\hline 14 & $\begin{array}{l}\text { La problématique n'est pas constatée } \\
\text { par les études antérieures ; }\end{array}$ & $30 \%$ & $30 \%$ & $40 \%$ & 2.8 & 1.690 \\
\hline
\end{tabular}




\begin{tabular}{|c|c|c|c|c|c|c|}
\hline \multirow[b]{2}{*}{$\mathbf{N}^{\circ}$} & \multirow[b]{2}{*}{ Items } & \multicolumn{3}{|c|}{ Pourcentage } & \multirow{2}{*}{ 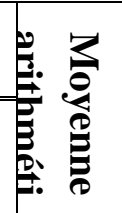 } & \multirow{2}{*}{ 常 } \\
\hline & & Oui & $\begin{array}{c}\text { Parf } \\
\text { ois }\end{array}$ & Non & & \\
\hline 15 & $\begin{array}{l}\text { La problématique n'est pas constatée } \\
\text { par une étude pilote ; }\end{array}$ & $30 \%$ & $30 \%$ & $40 \%$ & 2.8 & 1.690 \\
\hline 26 & $\begin{array}{l}\text { Ne pas rédiger une introduction pour } \\
\text { chaque axe de la recherche; }\end{array}$ & $30 \%$ & $30 \%$ & $40 \%$ & 2.8 & 1.690 \\
\hline 36 & $\begin{array}{l}\text { Faire beaucoup d'erreurs } \\
\text { d'orthographe ; }\end{array}$ & $30 \%$ & $30 \%$ & $40 \%$ & 2.8 & 1.690 \\
\hline 51 & $\begin{array}{l}\text { Ne pas citer les études antérieures les } \\
\text { plus pertinentes concernant le sujet } \\
\text { de la recherche ; }\end{array}$ & $30 \%$ & $30 \%$ & $40 \%$ & 2.8 & 1.690 \\
\hline 57 & $\begin{array}{l}\text { Ne pas déclarer la manière de choisir } \\
\text { l'échantillon de la recherche ; }\end{array}$ & $30 \%$ & $30 \%$ & $40 \%$ & 2.8 & 1.690 \\
\hline 4 & $\begin{array}{l}\text { Les variables de la recherche ne sont } \\
\text { pas déterminées dans le titre ; }\end{array}$ & $20 \%$ & $40 \%$ & $40 \%$ & 2.6 & 1.522 \\
\hline 38 & $\begin{array}{l}\text { Erreurs dans l'utilisation des } \\
\text { pronoms personnels et des pronoms } \\
\text { relatifs ; }\end{array}$ & $30 \%$ & $20 \%$ & $50 \%$ & 2.6 & 1.773 \\
\hline 18 & $\begin{array}{l}\text { Erreurs dans la rédaction des } \\
\text { hypothèses ; }\end{array}$ & $20 \%$ & $30 \%$ & $50 \%$ & 2.4 & 1.589 \\
\hline 28 & $\begin{array}{l}\text { Faire des citations sans citer la } \\
\text { référence (Faire un plagiat) ; }\end{array}$ & $20 \%$ & $30 \%$ & $50 \%$ & 2.4 & 1.589 \\
\hline 58 & $\begin{array}{l}\text { Utiliser des outils de collecte de } \\
\text { données inconvenables ; }\end{array}$ & $20 \%$ & $30 \%$ & $50 \%$ & 2.4 & 1.589 \\
\hline 62 & $\begin{array}{l}\text { Utiliser des méthodes statistiques } \\
\text { inappropriées ; }\end{array}$ & $20 \%$ & $30 \%$ & $50 \%$ & 2.4 & 1.589 \\
\hline 13 & $\begin{array}{l}\text { La problématique de la recherche } \\
\text { n'est pas clair; }\end{array}$ & $20 \%$ & $20 \%$ & $60 \%$ & 2.2 & 1.627 \\
\hline 17 & $\begin{array}{l}\text { Les questions de recherche ne sont } \\
\text { pas adéquates à la problématique ; }\end{array}$ & $20 \%$ & $20 \%$ & $60 \%$ & 2.2 & 1.627 \\
\hline 50 & $\begin{array}{llll}\text { Citer des études antérieures } & \text { ne } \\
\text { concernant pas le sujet de la } \\
\text { recherche; }\end{array}$ & $20 \%$ & $20 \%$ & $60 \%$ & 2.2 & 1.627 \\
\hline 71 & $\begin{array}{l}\text { Ne pas présenter des } \\
\text { convenables pour des } \\
\text { ultérieures ; }\end{array}$ & $20 \%$ & $20 \%$ & $60 \%$ & 2.2 & 1.627 \\
\hline 77 & $\begin{array}{l}\mathrm{Ne} \text { pas respecter les pratiques } \\
\text { reconnues dans son domaine ou son } \\
\text { milieu académique; }\end{array}$ & $20 \%$ & $20 \%$ & $60 \%$ & 2.2 & 1.627 \\
\hline 3 & Le titre ne montre pas précisément le & $10 \%$ & $30 \%$ & $60 \%$ & 2.0 & $\mathbf{1 . 3 6 5}$ \\
\hline
\end{tabular}




\begin{tabular}{|c|c|c|c|c|c|c|}
\hline \multirow[b]{2}{*}{$\mathbf{N}^{\circ}$} & \multirow[b]{2}{*}{ Items } & \multicolumn{3}{|c|}{ Pourcentage } & \multirow{2}{*}{ 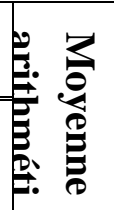 } & \multirow{2}{*}{ 远 } \\
\hline & & Oui & $\begin{array}{l}\text { Parf } \\
\text { ois }\end{array}$ & Non & & \\
\hline & but de la recherche ; & & & & & \\
\hline 25 & $\begin{array}{l}\text { Présenter des conceptions théoriques } \\
\text { n'ayant pas de relation avec la } \\
\text { problématique; }\end{array}$ & $10 \%$ & $30 \%$ & $60 \%$ & 2.0 & 1.365 \\
\hline 37 & $\begin{array}{l}\text { Erreurs dans l'utilisation } \\
\text { prépositions ; }\end{array}$ & $10 \%$ & $30 \%$ & $60 \%$ & 2.0 & 1.365 \\
\hline 53 & $\begin{array}{l}\text { Utiliser une méthodologie de } \\
\text { recherche inappropriée ; }\end{array}$ & $10 \%$ & $30 \%$ & $60 \%$ & 2.0 & 1.365 \\
\hline 54 & $\begin{array}{l}\text { Utiliser un design de recherche } \\
\text { inapproprié ; }\end{array}$ & $10 \%$ & $30 \%$ & $60 \%$ & 2.0 & 1.365 \\
\hline 2 & $\begin{array}{l}\text { Le titre ne détermine pas le domaine } \\
\text { de la recherche ; }\end{array}$ & $0 \%$ & $40 \%$ & $60 \%$ & 1.8 & .997 \\
\hline 45 & $\begin{array}{l}\text { Manque de l'objectivité à l'égard des } \\
\text { idées présentées ; }\end{array}$ & $10 \%$ & $20 \%$ & $70 \%$ & 1.8 & 1.349 \\
\hline 1 & $\begin{array}{l}\text { Le titre ne détermine pas précisément } \\
\text { le contenu de la recherche ; }\end{array}$ & $0 \%$ & $30 \%$ & $70 \%$ & 1.6 & .932 \\
\hline 5 & $\begin{array}{l}\text { Le titre ne précise pas la population } \\
\text { de la recherche ; }\end{array}$ & $0 \%$ & $30 \%$ & $70 \%$ & 1.6 & .932 \\
\hline 33 & $\begin{array}{l}\text { Utiliser la première personne du } \\
\text { singulier }(\mathrm{Je}) \text {; }\end{array}$ & $0 \%$ & $30 \%$ & $70 \%$ & 1.6 & .932 \\
\hline 55 & $\begin{array}{l}\text { Ne pas respecter les procédures de la } \\
\text { recherche scientifique ; }\end{array}$ & $0 \%$ & $30 \%$ & $70 \%$ & 1.6 & .932 \\
\hline 21 & $\begin{array}{l}\text { Ne pas présenter des définitions } \\
\text { opérationnelles pour la terminologie } \\
\text { de la recherche; }\end{array}$ & $0 \%$ & $20 \%$ & $80 \%$ & 1.4 & .814 \\
\hline 76 & $\begin{array}{l}\text { Ne pas respecter les normes, les règles } \\
\text { d'éthique et d'intégrité de la } \\
\text { recherche scientifique ; }\end{array}$ & $0 \%$ & $10 \%$ & $90 \%$ & 1.2 & .610 \\
\hline
\end{tabular}

Pour déterminer les erreurs communes de l'écriture académique chez les étudiants de magistère de la faculté de pédagogie (didactique du FLE), le chercheur classifie le degré de l'importance de l'erreur selon les proportions suivantes : $1: 2.5$ erreurs non communes, 2.6 : 3.5 erreurs communes à degré moyen, $3.6: 5$ erreurs communes.

Elaboration du test diagnostique :

Pour élaborer le test diagnostique, le chercheur a procédé comme suit :

1- Déterminer l'objectif du test ;

2- Description du test ;

3- Passation du test ; 
4- Choix de l'échantillon;

5- Résultats du test.

\section{Objectifs du test :}

Notre test vise essentiellement à :

1- Préciser les connaissances antérieures des étudiants de magistère de la faculté de pédagogie de Minia (didactique du FLE) sur l'écriture académique ;

2- Déterminer les erreurs communes de l'écriture académique chez les étudiants de magistère de la faculté de pédagogie de Minia (didactique du FLE).

\section{Sources du test :}

Le chercheur a choisi le contenu du test en se référant aux sources suivantes :

- Malo, Lise (2015) ;

- Service d'évaluation linguistique, Université TÉLUQ (2018);

- www.cafe.edu (2006) ;

- https://www.arealme.com/french-vocabulary-size-test/fr/

- https://www.bfmtv.com/culture/quiz-testez-votre-niveau-de-vocabulaire1399593.html

- https://candidat.hepl.ch/files/live/sites/systemsite/files/uer-fr/examen-op001/examenop001-sept-2012-qcm-corrigee-2012-hep-vaud.pdf

- https://fac.ksu.edu.sa/sites/default/files/2_39.pdf

- https://www.languagelevel.com/french/index.htm

- https://www.unige.ch/lettres/elcf/coursete/files/1014/1208/4495/certificat2011.pdf

- https://www.usj.edu.lb/pdf/testfr/test3.pdf

- https://www.victorias.fr/dossiers/tests/test-francais-fle-gratuit.html

\section{Description du test :}

Notre test comporte 6 questions : (note totale : 100 points)

\section{Première partie : Grammaire et Orthographe}

- Dans la $1^{\text {ère }}$ question, on demande aux participants de choisir la réponse correcte sur la grammaire et les structures ( 20 items $=20$ points $)$;

- Dans la $2^{\text {ème }}$ question, on demande aux participants de choisir les phrases correctement orthographiées ( 5 items $=5$ points);

\section{Deuxième partie : Syntaxe (structure des phrases - reformulation - connecteurs)}

- Dans la $1^{\text {ère }}$ question, on demande aux participants de choisir la phrase syntaxiquement correcte $(5$ items $=5$ points $)$;

- Dans la $2^{\text {ème }}$ question, on demande aux participants de reformuler les phrases données ( 5 items $=5$ points) ; 
- Dans la $3^{\text {ème }}$ question, on demande aux participants de relier les phrases avec des mots de connecteurs logiques $(15$ items $=15$ points $)$;

\section{Troisième partie : Rédaction d'un texte}

- Dans la $1^{\text {ère }}$ question, on demande aux participants d'écrire un texte contenant 7 paragraphes sur l'utilisation de l'internet dans l'éducation des élèves : progrès ou danger) en suivant la structure suivante : (50 points)

- Introduction : 5 points pour l'introduction bien formulée : définition du problème (sujet) qu'on va traiter, présentation de l'importance du sujet, présentation des idées principales du sujet (plan du développement), phrases correctes, ponctuation correcte) ;

- Développement (5 paragraphes) : 7 points pour chaque paragraphe bien formulé : idée unique, argumentation, cohérence avec le texte, enchainement avec le paragraphe précédent, utilisation des termes adéquats, phrases correctes, ponctuation correcte ;

- Conclusion (5 points : rappeler le déroulement de la réflexion - fournir une réponse claire au problème posé - rappeler les principaux points forts de son argumentation - phrases correctes, ponctuation correcte);

- Liste des références utilisées dans le texte : 5 références- un point pour chaque référence bien rédigée selon l'APA).

\section{Passation du test :}

Avant la passation du test, nous l'avons administré par une étude pilote (10 étudiants) pour calculer sa durée, sa validité et sa fidélité.

\section{La durée :}

Nous avons calculé la durée du test par la formule suivante :

Durée $=$ Temps total mis par chaque étudiant/Nombre des étudiants.

Durée $=1800 / 10=180$ minutes

Donc, la durée du test est 3 heures.

\section{Validité du test :}

Le chercheur a vérifié la validité du test par le calcul des corrélations question-total. Les corrélations des questions-total sont les suivantes :

$1^{\text {ère }}$ question : 0.38

$2^{\text {ème }}$ question : 0.77

$3^{\text {ème }}$ question : 0.43

$4^{\text {ème }}$ question : 0.64

$3^{\text {ème }}$ question : 0.49 
$6^{\text {ème }}$ question : 0.72

\section{La fidélité :}

Pour calculer la fidélité du test, nous avons appliqué le test deux fois, le sept Mars 2019, puis trois semaines après. Nous avons calculé le coefficient de corrélation entre les deux passations en appliquant la formule statistique suivante :

$$
r=\frac{\mathrm{n} \sum \mathrm{xy}-\left(\sum \mathrm{x}\right)\left(\sum \mathrm{y}\right)}{\sqrt{\left(\mathrm{n} \sum \mathrm{x}^{2}-\left(\sum \mathrm{x}\right)^{2}\right.}-\left(\mathrm{n} \sum \mathrm{y}^{2}-\left(\sum \mathrm{y}\right)^{2}\right.}
$$

$\mathrm{R}=$ coefficient de corrélation.

$\mathrm{N}=$ nombre des étudiants.

$\mathrm{T}=$ total des notes des étudiants.

$\mathrm{X}=$ notes de la $1^{\text {ère }}$ passation.

$\mathrm{Y}=$ notes de la $2^{\text {ème }}$ passation.

Indice de fidélité $=0,8$ ₹

\section{Résultats du test diagnostique :}

Notre test a été administré auprès d'un échantillon des étudiants de magistère de la faculté de pédagogie de Minia (10 étudiants) pendant le $2^{\mathrm{e}}$ semestre de l'année universitaire 2018/2019 (le 8 Avril 2019). Les résultats du test diagnostique ont montré que les étudiants de magistère de la faculté de pédagogie de Minia (didactique du FLE) ont les erreurs communes en écriture académique suivantes (le chercheur a considéré l'erreur faite par $70 \%$ ou plus de l'échantillon de la recherche comme erreur commune) :

- Erreurs dans la rédaction de l'introduction qui doit contenir les idées essentielles du sujet ;

- Erreurs dans la construction de la phrase (ordre des mots dans la phrase);

- Ecrire des phrases très longues ;

- Erreurs dans la reformulation en cas de la citation indirecte ;

- Ne pas respecter le principe «Une idée pour chaque paragraphe »;

- Rédiger des paragraphes très courts ou trop longs ;

- Erreurs dans l'utilisation du lexique et des expressions ;

- Utiliser des termes inadéquats pour le domaine ;

- Erreurs dans l'utilisation des connecteurs ;

- Faire beaucoup d'erreurs d'orthographe ;

- Erreurs dans l'utilisation de la ponctuation ;

- Ne pas utiliser le temps convenable ;

- Erreurs dans la conjugaison des verbes ;

- Erreurs dans l'utilisation des pronoms personnels et des pronoms relatifs ; 
- Erreurs dans les accords en genre et en nombre ;

- Concentration sur les citations et ne pas présenter ses propres idées ;

- Manque de l'argumentation qui soutient les idées présentées ;

- Manque de l'enchainement des idées dans le texte ;

- Manque de la cohérence dans le texte ;

- Traduire les idées littéralement de l'arabe en français ;

- Compter, essentiellement, sur des ressources secondaires ;

- Ne pas suivre une méthode convenable (APA) dans la rédaction des références ;

- Erreurs dans la rédaction des références (auteur, date, lieu de la publication, ...);

- Erreurs dans la rédaction de la conclusion qui doit fournir une réponse claire au problème posé.

\section{Elaboration du programme proposé :}

Notre étude vise à remédier aux erreurs de l'écriture académique chez les étudiants de magistère de la faculté de pédagogie de Minia (didactique du FLE). Pour atteindre ce but, nous élaborons un programme basé sur l'approche des processus d'écriture; pour élaborer notre programme, nous avons procédé comme suit :

- Définir l'objectif général du programme ;

- Définir les objectifs spécifiques du programme ;

- Déterminer le contenu du programme ;

- Définir les stratégies d'enseignement ;

- Déterminer les matériels du programme ;

- Déterminer les outils d'évaluation du programme.

\section{Objectif général :}

Le programme proposé vise à remédier aux erreurs communes de l'écriture académique chez les étudiants de magistère de la faculté de pédagogie de Minia (didactique du FLE).

\section{Objectifs spécifiques :}

Notre programme vise à remédier aux erreurs suivantes :

- Erreurs dans la rédaction de l'introduction qui doit contenir les idées essentielles du sujet ;

- Erreurs dans la construction de la phrase (ordre des mots dans la phrase);

- Ecrire des phrases très longues ;

- Erreurs dans la reformulation en cas de la citation indirecte ;

- Ne pas respecter le principe «Une idée pour chaque paragraphe »;

- Rédiger des paragraphes très courts ou trop longs ;

- Erreurs dans l'utilisation du lexique et des expressions ; 
- Utiliser des termes inadéquats pour le domaine ;

- Erreurs dans l'utilisation des connecteurs ;

- Faire beaucoup d'erreurs d'orthographe ;

- Erreurs dans l'utilisation de la ponctuation ;

- Ne pas utiliser le temps convenable ;

- Erreurs dans la conjugaison des verbes ;

- Erreurs dans l'utilisation des pronoms personnels et des pronoms relatifs ;

- Erreurs dans les accords en genre et en nombre ;

- Concentration sur les citations et ne pas présenter ses propres idées ;

- Manque de l'argumentation qui soutient les idées présentées ;

- Manque de l'enchainement des idées dans le texte ;

- Manque de la cohérence dans le texte ;

- Traduire les idées littéralement de l'arabe en français ;

- Compter, essentiellement, sur des ressources secondaires ;

- Ne pas suivre une méthode convenable (APA) dans la rédaction des références ;

- Erreurs dans la rédaction des références (auteur, date, lieu de la publication, ...);

- Erreurs dans la rédaction de la conclusion qui doit fournir une réponse claire au problème posé ;

\section{Contenu du programme :}

Notre programme se compose de 4 unités :

Unité 1. Les caractéristiques de l'écriture académique : Cette unité contient les sujets suivants :

- Caractéristiques de l'écriture académique ;

- Principes de la rédaction académique ;

- Le style de l'écriture académique.

Unité 2. Grammaire et Structure

Cette unité contient les sujets suivants :

- La phrase de sujet et les phrases de support ;

- Emploi des temps dans la thèse ;

- Les connecteurs ;

- La ponctuation.

Unité 3. Bibliographie de la recherche

Cette unité présente la manière de rédiger la liste des références selon l'APA.

Unité 4. Rédiger le rapport de la recherche

Cette unité contient les séquences suivantes : 
I. Génération des idées :

Dans cette partie de l'unité, le formateur doit stimuler la créativité des participants, les amener à réfléchir à la manière d'aborder le sujet d'écriture (chaque participant choisit un sujet selon son intérêt). Ensuite, ils déterminent les idées essentielles et les idées secondaires du sujet à travers la stratégie du remue-méninges. A ce stade, le plus important est le flux d'idées. Le formateur peut donner des conseils sur la manière d'améliorer leurs idées initiales.

\section{Lecture et prise des notes :}

Cette partie consiste à prendre note des informations et des idées que les étudiants trouvent dans les sources disponibles pour eux. Lorsqu'ils lisent une source, ils notent les informations importantes qu'ils lisent.

III. Faire un brouillon :

Ensuite, les participants organisent les notes autour des points principaux du sujet. Ils prennent des notes sur des photocopies ou des documents Internet imprimés en soulignant les informations importantes et en prenant des notes dans la marge. Puis, les participants écrivent des commentaires sur les informations ; les participants écrivent chaque information sur une carte avec le nom de l'auteur et les informations nécessaires sur la source. Les participants organisent les cartes autour des points principaux.

IV. Reformulation: dans cette partie, le formateur demande aux participants de paraphraser et reformuler les informations importantes dans leurs propres mots ; on leur demande, également, d'utiliser des guillemets pour les mots directement copiés.

V. Evaluation par les pairs : Les étudiants échangent leurs premières versions d'un texte et indiquent les modifications nécessaires (par exemple, une meilleure organisation, divisions de paragraphes, variété de phrases, choix de vocabulaire, correction grammaticale). Ils peuvent également agir en tant que rédacteurs en chef (repérer des répétitions de vocabulaire, des erreurs grammaticales, des fautes d'orthographe, etc.). Dans le même temps, les participants sont tenus de fournir des commentaires écrits aux auteurs étudiants.

VI. Evaluation par le formateur : L'évaluation, dans cette phase, est plutôt une évaluation qualitative. Les commentaires présentés par le formateur peuvent aider à renforcer la confiance des participants et à créer un sentiment positif. Les participants doivent rester favorables aux commentaires sur la correction grammaticale, lexicale ou syntaxique de leur travail. Afin de rendre cette activité interactive, on utilise un code de correction d'erreur, qui sert à mettre en évidence l'erreur mais qui oblige les participants à réfléchir quelle est l'erreur en réalité. Parfois, on pose des questions telles que Que voulez-vous dire ici? Ou Pouvez-vous m'en dire plus à propos de ça?

VII. Réécriture : Les brouillons sont renvoyés et les améliorations sont apportées en fonction des commentaires des pairs et du formateur. Ensuite, le projet final est écrit. En outre, les étudiants réécrivent le sujet en fonction des évaluations passées (évaluation par les pairs et évaluation du formateur). 


\section{L'expérimentation (l'enseignement) du programme :}

L'expérimentation du programme proposé passe par plusieurs étapes :

- Déterminer le design expérimental et sélectionner les participants ;

- Administrer le pré-test (le chercheur a utilisé le test diagnostique comme pré/post-test) ;

- Expérimenter le programme ;

- Administrer le post-test ;

- L'analyse des résultats ;

L'enseignement de notre programme a eu lieu durant les vacances d'été pendant les deux mois juillet et aout. Une période de 4 semaines a été consacrée à la mise en application de cette expérimentation. Un échantillon de dix participants des étudiants de magistère en didactique du FLE a formé le groupe expérimental. Notre programme a été soutenu par l'unité des stages à la faculté de pédagogie qui a organisé les séances et a préparé une salle pour l'exécution du programme. La partie pratique du programme a été exécutée dans la bibliothèque des études supérieures.

Dans la partie théorique (trois premières unités), le formateur présente aux participants les caractéristiques de l'écriture académique, les principes de la rédaction académique, le style de l'écriture académique, la phrase de sujet et les phrases de support, l'emploi des temps dans la thèse, les connecteurs et la manière de rédiger la liste des références selon l'APA.

Dans la partie pratique (unité 4. Rédiger le rapport de la recherche), chaque participant a choisi un sujet selon son intérêt. Le formateur a demandé aux participants de déterminer les idées essentielles à aborder dans leurs sujets. Après avoir déterminé les idées essentielles et les idées secondaires pour chaque sujet, les participants ont cherché dans la bibliothèque et sur l'internet pour trouver dans les références nécessaires pour leurs sujets. Lorsqu'ils lisaient une source, ils notaient les informations importantes. Ils prenaient des notes sur des photocopies imprimés en soulignant les informations importantes et en prenant des notes dans la marge. Puis, les participants écrivaient des commentaires sur les informations ; ils écrivaient chaque information sur une carte avec le nom de l'auteur et les informations nécessaires sur la source. Les participants organisaient les cartes autour des points principaux. Ensuite, le formateur demandait aux participants de paraphraser et reformuler les informations importantes dans leurs propres mots et d'utiliser des guillemets pour les mots directement copiés. Les écrits des participants étaient soumis à l'évaluation par les pairs et à l'évaluation du formateur. Pendant tous ces types d'évaluation, l'évaluateur mettait l'accent sur la détermination des erreurs communes par les participants. L'évaluation se passait à plusieurs reprises pour désigner les erreurs commises par les participants dans la syntaxe, la grammaire, la construction des paragraphes. Enfin, les participants réécrivaient le sujet en fonction des évaluations passées (évaluation par les pairs et évaluation du formateur).

Résultats de la recherche :

Après avoir fini l'expérimentation du programme proposé et administré le post-test de l'écriture académique en didactique du FLE sur le groupe de la recherche, le chercheur fait l'analyse statistique en utilisant le programme statistique SPSS (version 23) et il utilise "T" test pour calculer la moyenne arithmétique, l'écart type et la valeur de "t". 
Tableau 1 : La différence entre le pré-test et le post-test du groupe expérimental (Total)

\begin{tabular}{|c|c|c|c|c|c|c|}
\hline Test & $\mathrm{N}$ & MA & ET & $\mathrm{T}$ & S. ou N.S. & VI \\
\hline Pré-test (Total) & \multirow{2}{*}{10} & 45.1 & 3.78447 & \multirow{2}{*}{35.541} & \multirow{2}{*}{$\begin{array}{c}\text { Significative au } \\
\text { degré } 0.01 \mathrm{en} \\
\text { faveur du post-test }\end{array}$} & \multirow{2}{*}{0.99} \\
\hline $\begin{array}{c}\text { Post-test } \\
\text { (Total) }\end{array}$ & & 85.1 & 4.99889 & & & \\
\hline
\end{tabular}

$\mathrm{N}=$ nombre des étudiants $\mathrm{MA}=$ moyenne arithmétique $\quad \mathrm{ET}=$ écart type

$\mathrm{T}=$ valeur de "t" test $\quad \mathrm{S}=$ significative $\quad \mathrm{NS}=$ non significative

$\mathrm{VI}=$ valeur de l'influence

Nous avons calculé la moyenne arithmétique, l'écart type et la valeur de T. On remarque qu'il y a une différence significative entre la moyenne des notes $\mathrm{du}$ groupe expérimental au pré-test et celle au post-test (Total) en faveur du post-test. La valeur de T calculé est plus grande que celle du tableau au niveau de p>0,01. Nous avons, également, mesuré la valeur de l'influence qui est très élevée (0.99). Ce qui vérifie la vérité de l'hypothèse principale de la recherche : «Il y a une différence significative entre la moyenne des notes des étudiants du groupe expérimental au pré/post-test des erreurs communes de l'écriture académique chez les étudiants de magistère de la faculté de pédagogie de Minia (didactique du FLE) en faveur du post/test.» et ce qui montre l'efficacité du programme proposé pour remédier aux erreurs communes en écriture académique chez les étudiants de magistère de la faculté de pédagogie de Minia (didactique du FLE).

Tableau 2 : Les résultats du pré/post-test du groupe expérimental (Grammaire et orthographe)

\begin{tabular}{|c|c|c|c|c|c|c|}
\hline Test & $\mathrm{N}$ & MA & ET & $\mathrm{T}$ & S. ou N.S. & VI \\
\hline $\begin{array}{c}\text { Pré-test } \\
\text { (Grammaire et } \\
\text { orthographe) }\end{array}$ & \multirow[t]{2}{*}{10} & 11.5 & 1.50923 & \multirow{2}{*}{27.386} & \multirow{2}{*}{$\begin{array}{c}\text { Significative au } \\
\text { degré } 0.01 \text { en } \\
\text { faveur du post-test }\end{array}$} & \multirow{2}{*}{0.98} \\
\hline $\begin{array}{c}\text { Post-test } \\
\text { (Grammaire et } \\
\text { orthographe) }\end{array}$ & & 21.5 & 1.58114 & & & \\
\hline
\end{tabular}

Nous avons calculé la moyenne arithmétique, l'écart type et la valeur de T. On remarque qu'il y a une différence significative entre la moyenne des notes du groupe expérimental au pré-test et celle au post-test (Grammaire et Orthographe) en faveur du post-test. La valeur de $\mathrm{T}$ calculé est plus grande que celle du tableau au niveau de $\mathrm{p}>0,01$. Nous avons, également, mesuré la valeur de l'influence qui est très élevée (0.98). Ce qui vérifie la vérité de la première hypothèse secondaire de la recherche «Il y a une différence significative entre la moyenne des notes des étudiants du groupe expérimental au pré/post-test de l'écriture académique à l'égard de la grammaire et de l'orthographe chez les étudiants de magistère de la faculté de pédagogie de Minia (didactique du FLE) en faveur du post-test» et ce qui constate l'efficacité du programme proposé pour remédier aux erreurs communes en écriture académique à l'égard de la grammaire et de l'orthographe chez les étudiants de magistère de la faculté de pédagogie de Minia (didactique du FLE).

Tableau 3 : La différence entre le pré-test et le post-test du groupe expérimental (Syntaxe) 


\begin{tabular}{|c|c|c|c|c|c|c|}
\hline Test & $\mathrm{N}$ & MA & ET & $\mathrm{T}$ & S. ou N.S. & VI \\
\hline $\begin{array}{l}\text { Pré-test } \\
\text { (Syntaxe) }\end{array}$ & \multirow[t]{2}{*}{10} & 11.8 & 2.04396 & \multirow{2}{*}{20.226} & \multirow{2}{*}{$\begin{array}{c}\text { Significative au } \\
\text { degré } 0.01 \text { en } \\
\text { faveur du post-test }\end{array}$} & \multirow{2}{*}{0.97} \\
\hline $\begin{array}{l}\text { Post-test } \\
\text { (Syntaxe) }\end{array}$ & & 21.8 & 1.75119 & & & \\
\hline
\end{tabular}

Nous avons calculé la moyenne arithmétique, l'écart type et la valeur de T. On remarque qu'il y a une différence significative entre la moyenne des notes du groupe expérimental au pré-test et celle au post-test (Syntaxe) en faveur du post-test. La valeur de T calculé est plus grande que celle du tableau au niveau de $p>0,01$. Nous avons, également, mesuré la valeur de l'influence qui est très élevée (0.97). Ce qui vérifie la vérité de la deuxième hypothèse secondaire de la recherche : «Il y a une différence significative entre la moyenne des notes des étudiants du groupe expérimental au pré/post-test de l'écriture académique à l'égard de la syntaxe chez les étudiants de magistère à la faculté de pédagogie de Minia (didactique du FLE) en faveur du post-test» et ce qui montre l'efficacité du programme proposé pour remédier aux erreurs communes en écriture académique à l'égard de la syntaxe chez les étudiants de magistère de la faculté de pédagogie de Minia (didactique du FLE).

Tableau 4 : La différence entre le pré-test et le post-test du groupe expérimental (Rédaction d'un texte)

\begin{tabular}{c|c|c|c|c|c|c}
\hline \hline Test & $\mathrm{N}$ & $\mathrm{MA}$ & $\mathrm{ET}$ & $\mathrm{T}$ & S. ou N.S. & VI \\
\hline \hline $\begin{array}{c}\text { Pré-test } \\
\text { (Rédaction } \\
\text { d'un texte) }\end{array}$ & \multirow{2}{*}{10} & 21.8 & 1.87380 & & & \\
\cline { 1 - 1 } $\begin{array}{c}\text { Post-test } \\
\text { (Rédaction } \\
\text { d'un texte) }\end{array}$ & & 41.8 & 2.29976 & & $\begin{array}{c}\text { Significative au } \\
\text { degré } 0.01 \text { en } \\
\text { faveur du post-test }\end{array}$ & 0.98 \\
\cline { 3 - 4 } & & & & & \\
\hline
\end{tabular}

Nous avons calculé la moyenne arithmétique, l'écart type et la valeur de T. On remarque qu'il y a une différence significative entre la moyenne des notes du groupe expérimental au pré-test et celle au post-test (adéquation de la phrase) en faveur du post-test. La valeur de $\mathrm{T}$ calculé est plus grande que celle du tableau au niveau de p>0,01. De même, nous avons mesuré la valeur de l'influence qui est très élevée (0.98). Ce qui vérifie la vérité de la troisième hypothèse secondaire de la recherche : «Il y a une différence significative entre la moyenne des notes des étudiants du groupe expérimental au pré/post-test de l'écriture académique chez les étudiants de magistère de la faculté de pédagogie de Minia (didactique du FLE) en faveur du post/test.» et ce qui montre l'efficacité du programme proposé pour remédier aux erreurs communes en écriture académique chez les étudiants de magistère de la faculté de pédagogie de Minia (didactique du FLE).

Pour s'assurer de l'efficacité de notre programme proposé, nous avons, également, mesuré les résultats de l'expérimentation en utilisant le coefficient de Wilcoxon qui est non paramétrique et plus adéquat avec les groupes de petite taille, le tableau suivant montre ces résultats : 
Tableau 7 : Les résultats du pré/post-test par le coefficient de Wilcoxon

\begin{tabular}{r|l|l|l|l}
\hline \hline & $\begin{array}{c}\text { Grammaire et } \\
\text { orthographe }\end{array}$ & Syntaxe & $\begin{array}{c}\text { Rédaction d'un } \\
\text { texte }\end{array}$ & Total \\
\hline \hline $\mathrm{Z}$ & $-2.848-^{\mathrm{a}}$ & $-2.816^{\mathrm{a}}$ & $-2.810-^{\mathrm{a}}$ & $-2.810-^{\mathrm{a}}$ \\
\hline $\begin{array}{r}\text { Asymp. Sig. (2- } \\
\text { tailed) }\end{array}$ & .004 & .005 & .005 & .005 \\
\hline \hline
\end{tabular}

a. Basé sur des classements négatifs.

b. Test de classement de Wilcoxon

On remarque qu'il y a des différences significatives entre les moyennes des notes des participants au pré/post-test dans les trois parties du test, ainsi que le total en faveur du posttest (les moyennes les plus grandes). Ce qui met en valeur la progression due à l'utilisation du programme proposé. Ces résultats montrent l'efficacité du programme proposé basé sur l'approche des processus d'écriture dans la remédiation aux erreurs communes de l'écriture académique chez les étudiants de magistère (didactique du FLE).

\section{Discussion}

Les résultats de notre recherche montrent l'efficacité de l'approche des processus d'écriture pour remédier aux erreurs communes en écriture académique. Cette stratégie contient certains processus : la génération des idées, la prise des notes, la rédaction d'un brouillon, la reformulation, l'évaluation et la réécriture. Notre stratégie donne une grande importance aux processus de l'évaluation pour remédier aux erreurs communes de l'écriture académique. Le texte écrit par le participant est évalué plusieurs fois : une évaluation par les pairs et une évaluation faite par le formateur. Au début de l'expérimentation, une partie théorique sur les caractéristiques et les principes de l'écriture académique et son style a été enseignée. L'efficacité de l'approche des processus d'écriture dans la remédiation des erreurs communes de l'écriture académique peut être attribuable aux multiples processus que les participants exécutaient pour parvenir au texte dans sa forme finale : écrire, reformuler, évaluer et réécrire.

Les résultats de notre étude sont conformes avec ceux de l'étude de Sapkota (2013) qui ont indiqué que la technique de correction par les pairs et de correction des enseignants était productive dans l'enseignement de l'écriture. Les résultats de notre recherche sont, également, conformes à ceux de l'étude d'Omari (2005) qui ont indiqué l'efficacité de la méthode des processus d'écriture dans l'enseignement de l'écriture en anglais langue seconde chez les élèves de dixième année en Jordanie. De même, les résultats de notre recherche sont conformes avec ceux des études de Ho (2006), de Sun \& Feng (2009), de Bayat (2014) et d'Alodwan \& Ibnian (2014) qui ont montré l'efficacité de l'approche des processus d'écriture dans le développement des compétences de l'écriture. 


\section{Conclusion}

L'écriture académique est d'une importance primordiale pour tous les chercheurs dans tous les domaines surtout les chercheurs de magistère car ils sont en train de développer leurs compétences méthodologiques et discursives. Quand même, ceux-ci font beaucoup d'erreurs dans leurs écritures académiques. Ils ont, alors, besoin de remédier à ces difficultés. L'approche des processus d'écriture permet aux étudiants écrivains de réviser leurs écritures à plusieurs reprises à travers l'autoévaluation, l'évaluation par les pairs et l'évaluation par le formateur. La présente recherche a présenté une étude théorique sur les caractéristiques et le style de l'écriture académique, les étapes et les modèles de l'approche des processus d'écriture. La partie méthodologique de cette étude a porté sur la détermination des erreurs communes chez les étudiants de magistère (didactique du FLE) à travers un questionnaire adressé à un échantillon du corps enseignant en didactique du FLE. Cette étude a utilisé un test diagnostique pour déterminer les erreurs communes chez les étudiants de magistère de la faculté de pédagogie de Minia (didactique du FLE). Pour remédier aux erreurs communes en écriture académique chez les étudiants de magistère de la faculté de pédagogie de Minia (didactique du FLE), le chercheur a élaboré et expérimenté un programme basé sur l'approche des processus d'écriture ; l'expérimentation du programme a montré l'efficacité du programme proposé dans la remédiation aux erreurs communes chez les étudiants de magistère (didactique du FLE).

\section{Recommandations :}

A la lueur des résultats de cette étude, le chercheur présente les recommandations suivantes :

- Utiliser l'approche des processus d'écriture pour remédier aux erreurs communes de l'écriture académique chez les étudiants de magistère en didactique du FLE ;

- Favoriser l'évaluation par les pairs pour remédier aux erreurs communes de l'écriture académique chez les étudiants de magistère en didactique du FLE ;

- Entrainer, continuellement, les étudiants de magistère sur l'écriture académique pour être au courant des nouveautés dans ce domaine ;

- Entrainer les étudiants de magistère sur les étapes de la recherche académique pour produire des recherches de qualité ;

- Préparer et exécuter des ateliers pour développer les compétences linguistiques des étudiants de magistère et utiliser la langue pour des objectifs académiques.

\section{Suggestions :}

A la lueur des résultats de cette étude, le chercheur présente les suggestions suivantes :

- Vérifier l'efficacité de l'approche des processus d'écriture pour remédier aux erreurs communes de l'expression écrite en FLE chez les étudiants du cycle universitaire ;

- Vérifier l'efficacité de l'évaluation par les pairs pour remédier aux erreurs communes de l'expression orale en FLE chez les étudiants du cycle universitaire. 


\section{Références :}

Abas, Imelda Hermilinda and Abd Aziz, Noor Hashima (2016). Classification of 12writing process and writing strategies, Proceeding of ICECRS, 1 (2016) 367-380.

Adam, J.-M. (2005). La linguistique textuelle. Introduction à l'analyse textuelle des discours. Armand Colin, Paris.

Alodwan, Talal Abd Alhameed and Ibnian, Salem Saleh Khalaf (2014). The Effect of Using the Process Approach to Writing on Developing University Students' Essay Writing Skills in EFL, International Journal of Linguistics and Communication June 2014, Vol. 2, No. 2, pp. 147-163.

Al-Fadda, Hind (2012). Using wikis to facilitate group work and improve academic writing skills among university students, Study in curricula and methodology, $\mathrm{N}^{\circ} 178$, P. 1-21.

Allison, Desmond (2004). Creativity, students' academic writing, and EAP: exploring comments on writing in an English language degree programme, Journal of English for Academic Purposes 3(3):191-209 · July 2004, DOI: 10.1016/j.jeap.2003.11.005.

Alqiawi, Dalal Abdulla (2015). A Proposed Model On Developing Students' Academic Writing Based On Critical Thinking. International Interdisciplinary Journal of Education -December 2015, Volume 4, Issue 12, pp. 312-321.

Bailey, Stephen (2011). Academic Writing A Handbook for International Students, Third edition, Routledge, Taylor \& Francis Group, New York.

Barnett, Marva A. (1992). Writing as a Process, Northeast Conference. Récupéré de http://web.pdx.edu/ fischerw/courses/advanced/methods_docs/pdf_doc/wbf_c ollection/0001_0050/0009_Barnett_writing.PDF

Bayat, Nihat (2014).The Effect of the Process Writing Approach on Writing Success and Anxiety, Educational Sciences: Theory \& Practice, 14(3), p.1133-1141.

Boch, Françoise (2013). Former les doctorants à l'écriture de la thèse en exploitant les études descriptives de l'écrit scientifique, Linguagem em (Dis)curso, Tubarão, SC, v. 13, n. 3, p. 543-568, set./dez. 2013.

Bou, Paule, Cavalla, Cristelle (2011). Un référentiel-outil de compétences méthodologiques. L.Cadet, J.Goes JM.Mangiante. Langue(s) et intégration : Dimensions institutionnelle, socio-professionnelle et universitaire, Peter Lang, pp.365-381. $<$ hal-00699920>

Brown, H. Douglas (2001). Teaching by principles: An interactive approach to language pedagogy (2nd ed.). New York: Addison Wesley Longman.

Burnett, Leon (2008). How to improve your academic writing, University of Essex Printing Services@, Récupéré

de https://www.york.ac.uk/media/study/schoolsandcolleges/sixth-formresources/how-to-improve-your-academic-writing.pdf 
Charnock, Ross (1999). Les langues de spécialité et le langage technique: considérations didactiques. Reçu de https://journals.openedition.org/asp/2566.

Coffin, Caroline; Curry, Mary Jane; Goodman, Sharon; Hewings, Ann; Lillis, Theresa M. and Swann, Joan (2005). Teaching Academic Writing, Routledge, Taylor \& Francis Group: London and New York.

Donahue, C. (2008). Écrire à l'université : analyse comparée en France et aux Etats-Unis. Villeneuve d'Ascq : Presses Universitaires du Septentrion.

Ferraris, Christelle Annick \& Hernández, Clara Cecilia Uribe (2015). Écrire un article de recherche dans un contexte de licence en FLE. Synergies Mexique n ${ }^{\circ} 5$ - 2015 p. 127-138.

Guidère, Mathieu (2004). Méthodologie de la recherche, Guide du jeune chercheur en Lettres, Langues, Sciences humaines et sociales. Ellipses, ISBN 2. 7298-21767

Hart, Gail L. (2006). An analysis of student and instructor perceptions of students' writing skills at the university level, Ed.D., Florida International University, UMI Number: AAT3249705.

Hartley, James (2008). Academic Writing and Publishing, Routledge, Taylor \& Francis Group: London and New York.

Hayes, John (1996). A new framework for understanding cognition and affect in writing. In Levy, C. Michael \& Ransdell, Sarah (eds.) The science of writing: Theories, methods, individual differences, and applications. Mahwah, NJ: Lawrence Erbaum, New Jersey, 1-27.

Hayes, J. R. (2012). Modeling and remodeling writing. Written Communication, 29(3), 369388.

Hayes, John; Flower, Linda (1980) Identifying the organization of writing processes. In Gregg, Lee; Steinberg, Erwin (eds.) Cognitive processes in writing: An interdisciplinary approach. Hillsdale, NJ: Lawrence Erlbaum, 3-30.

Ho, Belinda (2006). Effectiveness of using the process approach to teach writing in six Hong Kong primary classrooms, Perspectives: Working Papers in English and Communication, 17(1) Spring 2006, pp. 1-52.

Ito, Tatsuya (2015). L'écriture académique et la pensée critique-Une méthodologie pour la pensée autonome. NU Ideas Volume 4.2. 2015. Nagoya University Institute of Liberal Arts \& Sciences. P. 57-63. Récupéré de http://nuideas.ilas.nagoyau.ac.jp/Volume4/4-4_Ito.pdf

Jubran, Serene Mousa (2017). A Program Based on the Integration of Product- Process Approach in Teaching Writing in Al-Balqa Applied University, Dirasat, Educational Sciences, Volume 44, No. 4, Supplement 8, pp. 361-376.

Maisonneuve, Hervé (2010). Guide pratique du thésard. Editions scientifique \& LC, Sanofi.

Malo, Lise (2015). Guide de préparation au test de français FEP, Récupéré de https://fep.umontreal.ca/documents/pdf/preparation_test_francais.pdf 
Mourlhon-Dallies, Florence (2011). Le français sur objectifs universitaires, entre français académique, français de spécialité et français pré-professionnel. Le Français sur Objectifs Universitaires - 2011 pp. 135-143.

Mroue, Mariam (2014). Ecrit de recherche universitaire : éléments pour une sensibilisation au positionnement scientifique à travers la phraséologie transdisciplinaire. Linguistique. Université de Grenoble. Français. 〈NNT : 2014GRENL021〉. $<$ tel-01321057>

Niño, Sandra Dolores Ruiz (2013). Working by projects: A way to enrich critical thinking and the writing process in a third grade EFL classroom, Colomb. Appl. Linguist. J., June - December 2013. Vol. 15 • Number 2 • Bogotá, Colombia. p. $205-220$.

Omari, Hamzah Ali (2005). The Effect of Using the Process Approach to Teach Writing to Basic Stage Students in Jordan on Their Writing Achievement, Dirasat, Educational Sciences, Volume 32, No. 1,

Onozawa, Chieko (2010). A Study of the Process Writing Approach, Mutual love school Maebashi international university review, No.10, p. 153-163.

Ouskourt, Mohammed (2008). Effectiveness of the Process Approach in Improving Writing: A Theoretical Background, Revue of Human Sciences, n³0, Décembre 2008Vol A, pp.69-75.

Paveau, M. et Sarfati, G. (2003). Les grandes théories de la linguistique. De la grammaire comparée à la pragmatique. Armand Colin, Paris.

Plakhotnik, Maria S. et Rocco, Tonette S. (2012). Implementing Writing Support Circles With Adult Learners in a Nonformal Education Setting: Priority, Practice, and Process. Journal of Adult Learning, Volume: 23 issue: 2, pp. 76-81.

Reuter, Yves (2004). Analyser les problèmes de l'écriture de recherche en formation. In: Pratiques : linguistique, littérature, didactique, $\mathrm{n}^{\circ} 121-122$. Normes et pratiques de l'écrit dans le supérieur. pp. 9-27;

Sapkota, Ashok (2012). Developing Students' Writing Skill through Peer and Teacher Correction: An Action Research. Journal of NELTA, Vol. 17 No. 1-2, December 2012, p. 70-82. DOI : 10.3126/nelta.v17i1-2.8094.

Service d'évaluation linguistique, Université TÉLUQ (2018). Test de français écrit SEL, Récupéré de www.teluq.ca/sel.

Smyth, T. R. (1996). Writing in psychology: A student guide. In Hartley, James (2008). Academic Writing and Publishing, Routledge, Taylor \& Francis Group : London and New York.

Sun, Chunling \& Feng, Guoping (2009). Process Approach to Teaching Writing Applied in Different Teaching Models, English Language Teaching, vol. 2, $\mathrm{n}^{\circ}$ 1. P. 150155.

Sydney University (2019). Types of academic writing. Received from https://sydney.edu.au/students/writing/types-of-academic-writing.html 
Whitaker, Anne (2009). Academic writing guide 2010, City university of Seattle, Bratislava, Slovakia

http://www.vsm.sk/Curriculum/academicsupport/academicwritingguide.pdf .

White, R. and Arndt, V. (1991). Process Writing. Essex: Addison Wesley Longman Ltd.

www.cafe.edu (2006). Écrire de la dissertation à l'œuvre littéraire. Récupéré de http://cafe.edu/redaction/fra1013/mod02.html

Zabo, Idrissa Assumani (2017). Je développe mes compétences en écriture scientifique. Reçu de https://www.researchgate.net/publication/316855883<

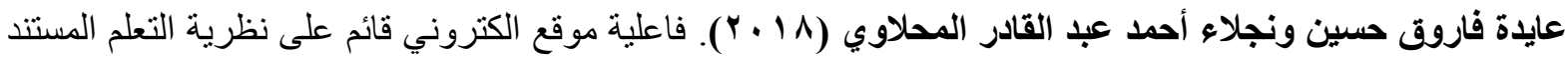

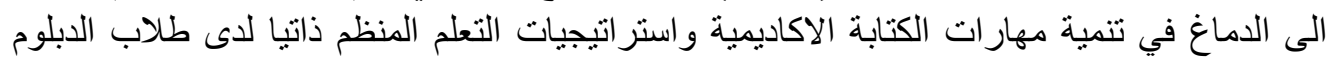

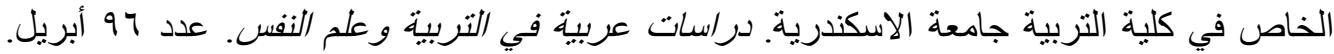

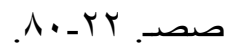




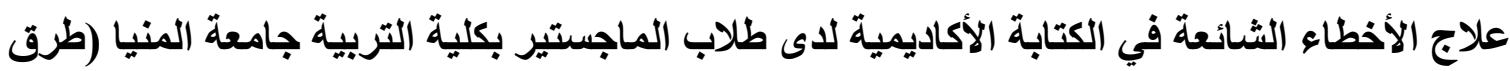

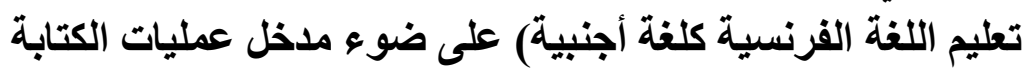

$$
\text { محمد جمعة رفاعي إبراهيم }
$$

مدرس المناهج وطرق التدريس ـ كلية التربية - جامعة المنيا

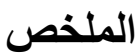

الكتابة الأكاديمية هي وسيلة لعرض الدراسات ونتائج البحوث العلمية في جميع مجالات العلوم. هذه الكتابة لها خصائصها و أسلوبها المحدد. ويحتاج طلاب الجامعة دائمًا إلى تحسين مهار اتهم في الكتابة الأكاديمية وتصحيح أخطائهم في هذا المجال. وهذا البحث يهدف إلى تحديد الأخطاء الثائعة في الكتابة الأكاديمية بين طلاب الماجستير تخصص طرق تدريس اللغة الفرنسية كلغة أجنبية. ولتحقيق هذا الهدف،

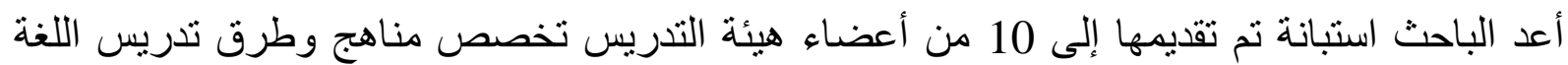

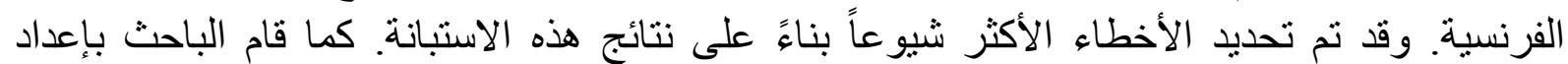

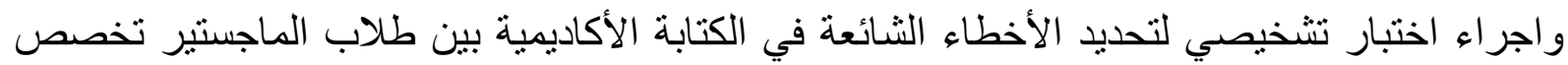

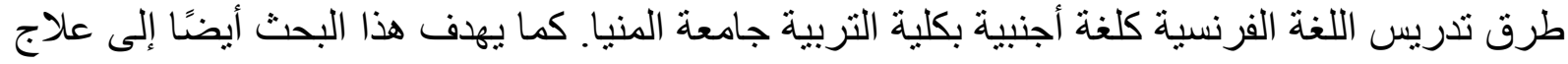
الأخطاء الثنائعة في الكتابة الأكاديمية بين طلاب الماجستير تخصص طاجية طرق تدريس اللغة الفرنسية كلغة

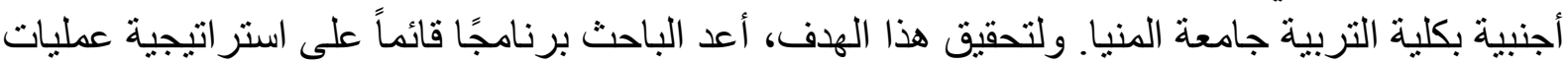

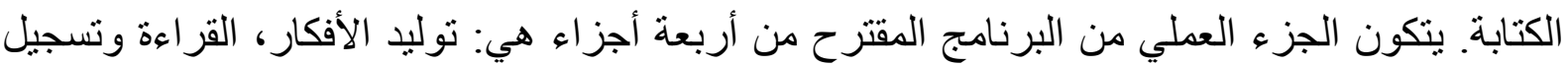

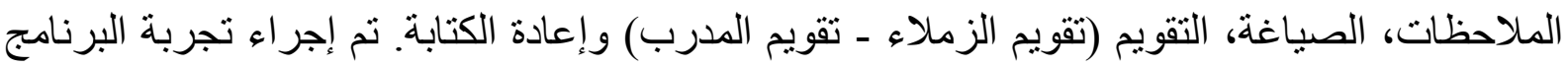
المقترح خلال العطلة الصيفية خلال شهري يوليو وأغسطس لدى مجموعة من طلاب الماجستير لئه

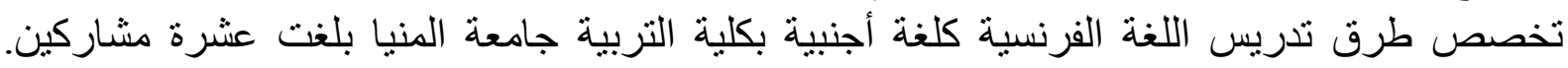
ولقياس فاعلية البرنامج قام الباحث بتطبيق اختبار قبلي/بعدي في الكتابة الأكاديمية قبل وبعد تطبيق

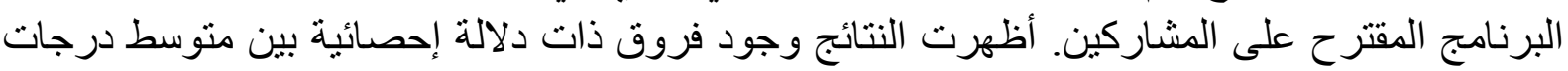

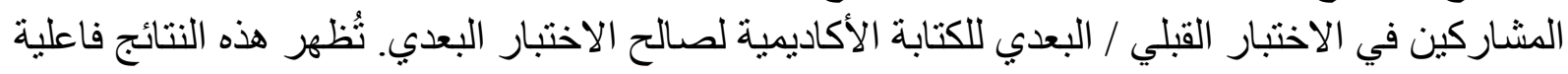
البرنامج المقترح القائم على استراتيجية عمليات الكتابة في علاج الأخطاء الثائعة في الكتابة الأكاديمية

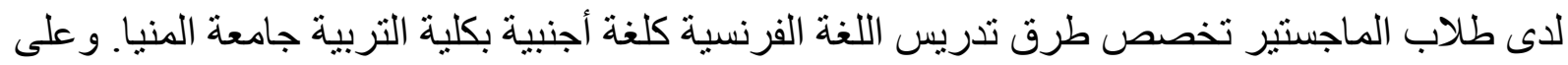
ذلك يوصي الباحث باستخدام استر اتيجية عمليات الكتابة في تدريس الكتابة الأكاديمية لطلاب الدربة لاستات

كلمات مفتاحية: الكتابة الأكاديمية ـ مدخل عمليات الكتابة ـ الأخطاء الثائعة ـ طرق تعليم اللغة الفرنسية كلغة أجنبية 